\title{
CATEGORICAL LENGTH, RELATIVE L-S CATEGORY AND HIGHER HOPF INVARIANTS
}

\author{
NORIO IWASE \\ Faculty of Mathematics, Kyushu University \\ Fukuoka 810-8560, Japan \\ E-mail: iwase@math.kyushu-u.ac.jp
}

\begin{abstract}
In this paper we introduce the categorical length, a homotopy version of Fox categorical sequence, and an extended version of relative L-S category which contains the classical notions of Berstein-Ganea and Fadell-Husseini. We then show that, for a space or a pair, the categorical length for categorical sequences is precisely the L-S category or the relative L-S category in the sense of Fadell-Husseini respectively. Higher Hopf invariants, cup length, module weights, and recent computations by Kono and the author are also studied within this unified L-S theory based on the categorical length of categorical sequences.
\end{abstract}

1. Introduction. Throughout this paper, we work in $\mathcal{T}$ the category of topological spaces and maps, or the category of pairs $\mathcal{T}^{A}$ in which an object is a pair $(X: A)$ with an inclusion $i^{X}: A \hookrightarrow X$ and a morphism is a map of pairs $f:(X: A) \rightarrow(Y: A)$ with $i^{Y}=f \circ i^{X}$. A closed subset is always assumed to be a neighbourhood deformation retract, and a pair is assumed to be an NDR-pair in the sense of G. Whitehead [29]. The one-point-space is denoted by $*$. The (normalised) Lusternik-Schnirelmann category $\operatorname{cat}(X), \mathrm{L}-\mathrm{S}$ category for short, is introduced in [22] as the least number $m$ such that there is a covering of $X$ by $m+1$ closed subsets $U_{j}, 0 \leq j \leq m$, where each $U_{j}$ is contractible in $X$. By modifying the idea due to R. Fox [8], T. Ganea [9] gives the following definition of a strong version of L-S category for a space $X$ : the strong L-S category Cat $(X)$ is the least number $m$ such that there is a space $Y \simeq X$ with a covering of $Y$ by $m+1$ closed subsets $U_{j}, 0 \leq j \leq m$ where each $U_{j}$ is contractible in itself. By Ganea [9], it is shown that

$$
\operatorname{cat}(X) \leq \operatorname{Cat}(X) \leq \operatorname{cat}(X)+1
$$

2000 Mathematics Subject Classification: Primary 55M30; Secondary 55Q25.

Key words and phrases: Lusternik-Schnirelmann category, categorical sequence, Hopf invariant, cone decomposition.

The paper is in final form and no version of it will be published elsewhere. 
REMARK 1.1. Fadell and Husseini [7] introduced a notion of relative L-S category as follows: for a pair $(K: A)$, cat $^{\mathrm{FH}}(K, A)$ is given as the least number $m$ such that there is a covering of $K$ by $m+1$ closed subsets $V \supset A$ and $U_{j}, 1 \leq j \leq m$ where $V$ is compressible relative $A$ into $A$ in $K$ and each $U_{j}$ is contractible in $K$. It is also clear by definition that $\operatorname{cat}^{\mathrm{FH}}(K, *)=\operatorname{cat}(K)$.

By G. Whitehead [29], the definition of L-S category is interpreted in terms of deformation of a diagonal map as the following definition for a space $X$.

Definition 1.2. The L-S category $\operatorname{cat}(X)$ of $X$ is the least number $m$ such that the $m+1$ fold diagonal map $\Delta^{m+1}: X \rightarrow \prod^{m+1} X$ is compressible into the fat wedge $\mathrm{T}^{m+1} X=$ $\left\{\left(x_{0}, x_{1}, \ldots, x_{m}\right) \in \prod^{m+1} X \mid \exists i x_{i}=*\right\} \subseteq \prod^{m+1} X$.

Similarly to the above, one can give an alternative definition of a relative L-S category in the sense of Fadell and Husseini [7] for a pair $(K: A)$ to fit in with Whitehead's definition of L-S category.

Definition 1.3. Let $A \subseteq K$. Then the L-S category cat ${ }^{\mathrm{FH}}(K, A)$ is the least number $m \geq 0$ such that the $m+1$ fold diagonal map $\Delta_{K}^{m+1}: K \rightarrow \prod^{m+1} K$ is compressible relative $A$ into the fat wedge $\mathrm{T}^{m+1}(K: A)=A \times \prod^{m} K \cup K \times \mathrm{T}^{m} K \subseteq \prod^{m+1} K$ of a pair $(K: A)$.

REMARK 1.4. For any map $f: A \rightarrow K$, we may assume that $f$ is an inclusion up to homotopy, and hence the definition of relative L-S category implies a definition of cat $^{\mathrm{FH}}(f)$ the L-S category of $f$ in the sense of Fadell and Husseini.

In the present paper, we alter the Fox's definition of a categorical sequence to fit in with Whitehead's definition of L-S category:

Definition 1.5. A categorical sequence for a space $X$ is a sequence of closed subspaces $F_{0} \subset \cdots \subset F_{i} \subset \cdots \subset F_{m}$ such that $F_{m} \simeq X, F_{0} \simeq *$ in $X$ and $\Delta_{i}: F_{i} \stackrel{\Delta}{\rightarrow} F_{i} \times F_{i} \subset$ $F_{m} \times F_{m}$ is compressible into $F_{i-1} \times F_{m} \cup F_{m} \times *$ relative $F_{i-1}$ for any $i>0$, where we identify $F_{i-1}$ with its diagonal image in $F_{i-1} \times F_{i-1} \subset F_{i-1} \times F_{m} \cup F_{m} \times *$. Let us call the least such $m \geq 0$ the 'categorical length' of $X$ and denote it by catlen $(X)$.

Inspired by the definition of a relative L-S category due to Fadell and Husseini, we introduce a relative version of categorical sequence:

Definition 1.6. A categorical sequence for a pair $(X: A)$ is a sequence of pairs $\left(F_{0}: A\right) \subset$ $\cdots \subset\left(F_{i}: A\right) \subset \cdots \subset\left(F_{m}: A\right)$ such that $\left(F_{m}: A\right) \simeq(X: A)$ relative $A, F_{0} \simeq A$ relative $A$ in $X$ and $\Delta_{i}: F_{i} \stackrel{\Delta}{\rightarrow} F_{i} \times F_{i} \subset F_{m} \times F_{m}$ is compressible into $F_{i-1} \times F_{m} \cup F_{m} \times A$ relative $F_{i-1}$, $i>0$. Let us call the least such $m \geq 0$ the 'categorical length' of a space $X$ relative to $A$ and denote it by catlen $(X: A)$.

To describe the categorical sequence in terms of a relative L-S category, we give a definition of a new extended version of relative L-S category: from now on, we work in the category $\mathcal{T}^{A}$. We remark that, if $A=*$ the one point space, then $\mathcal{T}^{A}$ is the usual category of based connected spaces and based maps. We say that $(X, K: A)$ is a pair in $\mathcal{T}^{A}$ when $(X: A)$ and $(K: A)$ are objects in $\mathcal{T}^{A}$ and $(X, K)$ is a pair in $\mathcal{T}$, that $(X, K, L: A)$ is a triple in $\mathcal{T}^{A}$ when $(X: A),(K: A),(L: A)$ are objects in $\mathcal{T}^{A}$ and $(X, K, L)$ is a triple 
in $\mathcal{T}$, and that $(X ; K, L: A)$ is a triad in $\mathcal{T}^{A}$ when $(X: A),(K: A),(L: A)$ are objects in $\mathcal{T}^{A}$ and $(X ; K, L)$ is a triad in $\mathcal{T}$.

We remark, for any pair $(X, K: A)$ in $\mathcal{T}^{A}$, that the diagonal image of $A$ in $\prod^{m+1} X$ is in the subspace $\mathrm{T}^{m+1}(X, L)$. Thus for any $(X: A) \supset(L: A) \in \mathcal{T}^{A}$, we regard $\left(\prod^{m+1} X: A\right) \supset$ $\left(\mathrm{T}^{m+1}(X, L): A\right) \in \mathcal{T}^{A}$.

Definition 1.7. Let $(X ; K, L: A)$ be a triad in $\mathcal{T}^{A}$. Then $\operatorname{cat}(X ; K, L: A)$ is the least number $m$ such that the restriction of the $m+1$ fold diagonal map of $X$ to $K,\left.\Delta^{m+1}\right|_{K}$ : $K \rightarrow \prod^{m+1} X$, is compressible relative $A$ into $\mathrm{T}^{m+1}(X, L)$.

Using Harper's arguments on the homotopy of maps to the total space of a fibration in [12], Cornea [4] has given a proof of the following:

Proposition 1.8. Let $(X: A)$ be an object in $\mathcal{T}^{A},(Y, K: A)$ be a pair in $\mathcal{T}^{A}$ with the inclusion $j:(K: A) \hookrightarrow(Y: A)$ and $f:(X: A) \rightarrow(Y: A)$ be a map in $\mathcal{T}^{A}$. If $\left.f\right|_{X}: X \rightarrow Y$ has a compression $\sigma: X \rightarrow K$ such that $j \circ \sigma \sim f$ and $\sigma \circ i^{X} \sim i^{K}$ in $\mathcal{T}$, then there is a map $\sigma^{\prime}:(X: A) \rightarrow(K: A)$ a compression relative $A$ of $f$ such that $\left.\sigma \sim \sigma^{\prime}\right|_{X}: X \rightarrow K$.

One of its direct consequences is:

Corollary 1.9. Let $(X ; K . L: A)$ be a triple in $\mathcal{T}^{A}$. Then $\operatorname{cat}(X ; K, L: A)$ is the same as the least number $m$ such that $\left.\Delta^{m+1}\right|_{K}: K \rightarrow \prod^{m+1} X$ is compressible to a map $s:$ $K \rightarrow \mathrm{T}^{m+1}(X, L)$ such that $\left.s\right|_{A}$ is homotopic to the diagonal map $\Delta_{A}: A \rightarrow \prod^{m+1} A \subset$ $\mathrm{T}^{m+1}(X, L)$.

REMARK 1.10. (1) $\operatorname{cat}(X ; X, *: *)=\operatorname{cat}(X)$ and $\operatorname{cat}(X ; *, *: *)=0$.

(2) We denote $(X ; X, L: A)$ by $(X, L: A),(X ; K, A: A)$ by $(X ; K: A),(X ; X: A)$ by $(X: A)$, $(X ; K, L: *)$ by $(X ; K, L),(X ; K, *)$ by $(X ; K)$ and $(X: *)=(X ; X, *: *)=(X ; X)$ by $X$.

(3) We may replace inclusions $(L: A) \hookrightarrow(X: A)$ and $(K: A) \hookrightarrow(X: A)$ by maps $f$ : $(L: A) \rightarrow(X: A)$ and $g:(K: A) \rightarrow(X: A)$ in $\mathcal{T}^{A}$, since every such map is an inclusion map up to homotopy relative $A$ by taking the mapping cylinder of $K \cup_{A} L \stackrel{g \cup_{A} f}{\longrightarrow}$ $X$. Then we often denote $\operatorname{cat}(X ; K, L: A)$ by $\operatorname{cat}(g, f)$. By applying (1), we have $\operatorname{cat}(g, *)=\operatorname{cat}(g)$.

There is another classical notion of relative L-S category due to Berstein and Ganea [2].

Definition 1.11. Let $K \subset X$. Then the L-S category cat ${ }^{\mathrm{BG}}(X, K)$ is the least number $m \geq 0$ such that restriction to $K$ of the $m+1$ fold diagonal map $\Delta_{X}^{m+1}: X \rightarrow \prod^{m+1} X$ is compressible into the fat wedge $\mathrm{T}^{m+1} X$.

REMARK 1.12. For any map $f: K \rightarrow X$, we may assume that $f$ is an inclusion up to homotopy, and hence the above definition of the L-S category implies a definition of cat $^{\mathrm{BG}}(f)$ the L-S category of $f$ in the sense of Berstein and Ganea.

Arkowitz and Lupton [1] have also defined their relative L-S category for a map $h: X \rightarrow Y$. Since a map is up to homotopy a fibration, we may assume that $h$ is a fibration with fibre $L=h^{-1}(*) \subset X$. Then the relative L-S category of $h$ in the sense of Arkowitz and Lupton depends only on the pair $(X, L)$ by its definition. 
Definition 1.13. Let $L \subset X$. Then the L-S category cat ${ }^{\mathrm{AL}}(X, L)$ is the least number $m \geq 0$ such that the $m+1$ fold diagonal map $\Delta_{X}^{m+1}: X \rightarrow \prod^{m+1} X$ is compressible into the fat wedge $\mathrm{T}^{m+1}(X, L)$.

Then we prove:

THEOREM 1.14. The known three relative $L$-S categories are special cases of our new relative $L-S$ category:

(1) Let $X=K \supset L=A \supset *$. Then $\operatorname{cat}(X: A)=\operatorname{cat}(X ; X, A: A)=\operatorname{cat}^{\mathrm{FH}}(X, A)$.

(2) Let $X \supset K \supset L=A=*$. Then $\operatorname{cat}(X ; K)=\operatorname{cat}(X ; K, *: *)=\operatorname{cat}^{\mathrm{BG}}(X, K)$. More generally for a map $g: K \rightarrow X$ in $\mathcal{T}_{*}$, we have $\operatorname{cat}(g, *)=\operatorname{cat}^{\mathrm{BG}}(g)$.

(3) Let $K=X \supset L \supset A=*$. Then $\operatorname{cat}(X, L)=\operatorname{cat}(X ; X, L: *)=\operatorname{cat}^{\mathrm{AL}}(X, L)$.

We also introduce a new higher Hopf invariant: let $(X ; K, L: A)$ be a triad in $\mathcal{T}^{A}$, let $V$ be a co-loop co-H-space, i.e., a one-point-union of a 1-connected co-H-space with finitely-many circles, and let $\alpha: V \rightarrow K$ be a map in $\mathcal{T}$ such that $X \supset \hat{K}=K \cup_{\alpha} C V \supset K$. If $\operatorname{cat}(X ; K, L: A) \leq m$, then a relative higher Hopf invariant $H_{m}^{(X ; K, L: A)}(\alpha)$ is defined as a subset of $[V, \Omega(X, L) * \Omega(X) * \cdots * \cdots(X)]$. If $K \supset L$ and $\operatorname{cat}(K ; K, L: A) \leq m$, then an absolute higher Hopf invariant $H_{m}^{(K, L: A)}(\alpha)$ is defined as a subset of $[V, \Omega(K, L) * \Omega(K) *$ $\cdots * \Omega(K)]$ (see $\S 4$ for more details). The following result clarifies how a higher Hopf invariant determines whether a cone decomposition reduces to a categorical sequence or not.

TheOREM 1.15. Let $(X ; K, L: A)$ be a triad in $\mathcal{T}^{A}$, let $V$ be a co-loop co-H-space and let $\alpha: V \rightarrow K$ be a map in $\mathcal{T}$ such that $X \supset \hat{K}=K \cup_{\alpha} C V \supset K$. If $\operatorname{cat}(X ; K, L: A) \leq m$ and $H_{m}^{(X ; K, L: A)}(\alpha)=0$, then $\operatorname{cat}(X ; \hat{K}, L: A) \leq m$.

From now on, we abbreviate $H_{m}^{(X ; K, A: A)}(\alpha)$ by $H_{m}^{(X ; K: A)}(\alpha), H_{m}^{(X ; K, *)}(\alpha)$ by $H_{m}^{(X ; K)}(\alpha), H_{m}^{(K, A: A)}(\alpha)$ by $H_{m}^{(K: A)}(\alpha)$ and $H_{m}^{(K, *)}(\alpha)$ by $H_{m}^{K}(\alpha)$. Note that the definition of the absolute higher Hopf invariant $H_{m}^{K}(\alpha)$ coincides with the ordinary definition of the higher Hopf invariant $H_{m}(\alpha)$ in the sense of [14].

The main goal of this paper is to proof:

Theorem 1.16. For any $X$ in $\mathcal{T}$, we have cat $(X)=$ catlen $(X)$. More generally, for any object $(X: A) \in \mathcal{T}^{A}$, we have catlen $(X: A)=\operatorname{cat}(X: A)=\operatorname{cat}^{\mathrm{FH}}(X, A)$.

Corollary 1.17. Let $(X: A)$ be an object in $\mathcal{T}^{A}$. If cat ${ }^{\mathrm{FH}}(X, A)=m>0$, then there exists a sequence of pairs $\left\{\left(F_{i}: A\right) ; 0 \leq i \leq m\right\}$ such that $\left(F_{0}: A\right) \simeq(A: A)$ in $\left(F_{m}: A\right),\left(F_{m}: A\right) \simeq$ $(X: A)$ relative $A$ and $\operatorname{cat}\left(X ; F_{i}: A\right) \leq i, i>0$. Moreover, $\operatorname{cat}\left(F_{m} / F_{i-1} ; F_{i} / F_{i-1}\right) \leq 1$ with a partial co-action $F_{i} \rightarrow F_{m} / F_{i-1} \vee F_{m}$ along the collapsing map $F_{i} \rightarrow F_{i} / F_{i-1} \subseteq$ $F_{m} / F_{i-1}, i>0$. In particular, $F_{m} / F_{m-1}$ is a co-H-space co-acting on $F_{m}$ along the collapsing map $F_{m} \rightarrow F_{m} / F_{m-1}$.

2. $A_{\infty}$-decomposition of a map. In [9], Ganea introduced a so-called 'fibre-cofibre' construction for a map, which can be interpreted as the pullback construction from the view-point of Definition 1.3. We may regard this construction as an $A_{\infty}$-decomposition of a map using the pushout-pullback diagram (see [13, Lemma 2.1] and also Sakai [24] for the detailed proof in a general context): 


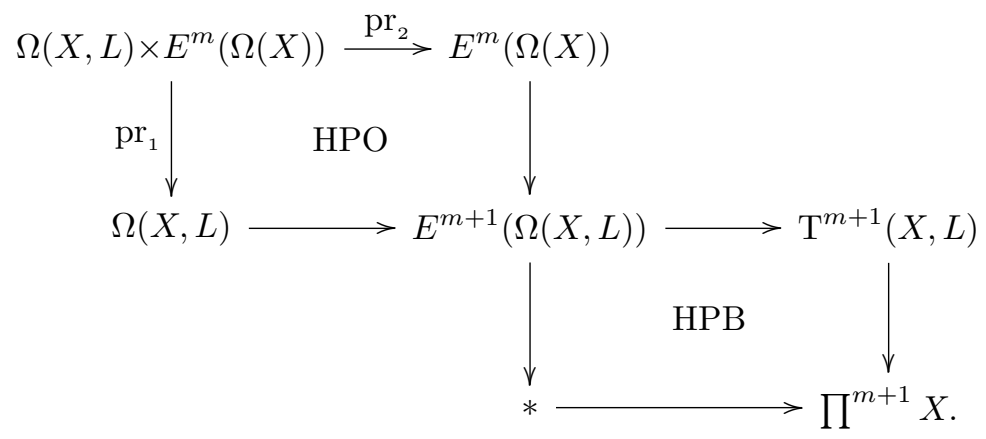

Let us recall that, in $\mathcal{T}$, the homotopy fibre of $\mathrm{T}_{i=0}^{m}\left(X, A_{i}\right) \hookrightarrow \prod^{m+1} X$ has the homotopy type of the join $\Omega\left(X, A_{0}\right) *^{m+1} * \Omega\left(X, A_{m}\right)$. Let $(X ; K, L: A)$ be a triad in $\mathcal{T}^{A}$ and write $E^{m}(\Omega(X))=\Omega(X) * \cdots * \Omega(X)$ which has the homotopy type of the homotopy fibre of $\mathrm{T}^{m}(X, *) \hookrightarrow \prod^{m} X$. The homotopy fibre of the inclusion $\mathrm{T}^{m+1}(X, L)$ $\hookrightarrow \prod^{m+1} X$ has the homotopy type of $E^{m+1}(\Omega(X, L))=\Omega(X, L) * \Omega(X) * \cdots * \Omega(X)$ : consider the homotopy pushout-pullback diagram in $\mathcal{T}$, which is given by [13, Lemma 2.1$]$ with $(Y, B)=\left(\prod^{m} X, \mathrm{~T}^{m} X\right), Z=*$ and $f=g=*$. Thus we see that the homotopy fibre of the inclusion $\mathrm{T}^{m+1}(X, L) \hookrightarrow \prod^{m+1} X$ has the homotopy type of $\Omega(X, L) * E^{m}(\Omega(X))=$ $E^{m+1}(\Omega(X, L))$ by induction.

Similarly, we define $P^{m}(\Omega(X, L))$ inductively from $P^{0}(\Omega(X, L))=L$ as the homotopy pushout in the following homotopy pushout-pullback diagram which is given by [13, Lemma 2.1] with $(Y, B)=\left(\prod^{m} X, \mathrm{~T}^{m} X\right), Z=X$ and $(f, g)=\left(1_{X}, \Delta_{X}^{m}\right)$ :

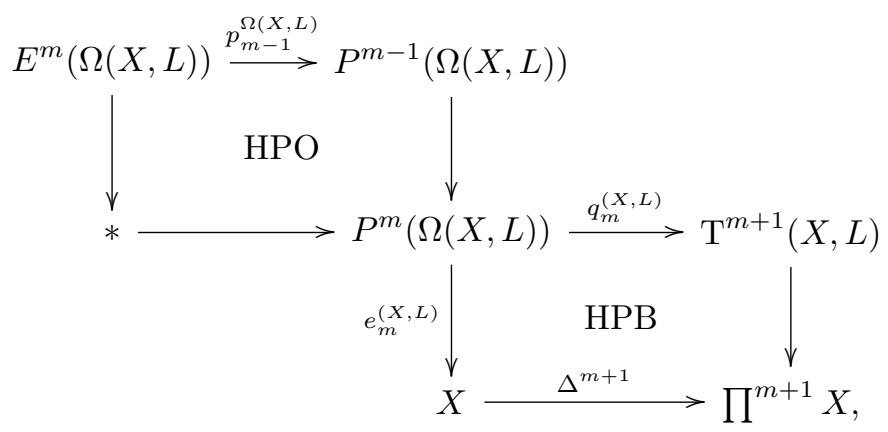

where $q_{m}^{(X, L)}$ covers the diagonal map $\Delta^{m+1}: X \rightarrow \prod^{m+1} X$. Then we define $p_{m+1}^{\Omega(X, L)}$ : $E^{m+1}(\Omega(X, L)) \rightarrow P^{m}(\Omega(X, L))$ as the homotopy fibre of $e_{m}^{(X, L)}: P^{m}(\Omega(X, L)) \rightarrow X$ given in the diagram, where $e_{0}^{(X, L)}: L \hookrightarrow X$ is just the canonical inclusion. These constructions due to Ganea [9] yield the following ladder of fibrations which have the same fibre $\Omega(X)$, giving a generalisation of an $A_{\infty}$-structure (see Stasheff [25]):

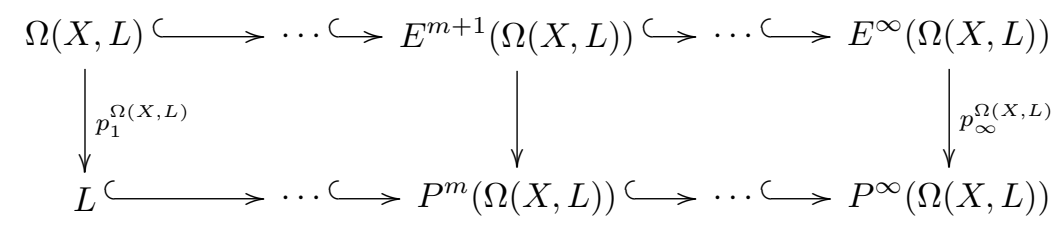


together with $e_{\infty}^{(X, L)}: P^{\infty}(\Omega(X, L))=\bigcup_{m} P^{m}(\Omega(X, L)) \rightarrow X$ given by $\left.e_{\infty}^{(X, L)}\right|_{P^{m}(\Omega(X, L))}$ $=e_{m}^{(X, L)}$ with fibre $E^{\infty}(\Omega(X, L))$, where the upper horizontal arrows are null-homotopic. Since $E^{\infty}(\Omega(X, L))=\bigcup_{m} E^{m}(\Omega(X, L))$ is weakly contractible, $e_{\infty}^{(X, L)}: P^{\infty}(\Omega(X, L))=$ $\bigcup_{m} P^{m}(\Omega(X, L)) \rightarrow X$ is a week equivalence. If further $X$ is a CW complex, then there is a right homotopy inverse $h^{(X, L)}: X \rightarrow P^{\infty}(\Omega(X, L))$ of $e_{\infty}^{(X, L)}$, where $h^{(X, L)}$ is also a weak equivalence.

The ladder (2.3) is natural with respect to a map of triads in $\mathcal{T}^{A}$ :

Lemma 2.1. For any map $f:(X ; K, L: A) \rightarrow\left(X^{\prime} ; K^{\prime}, L^{\prime}: A\right)$ of triads in $\mathcal{T}^{A}$, there is the following commutative diagram with $\left.f\right|_{(X, L)}:(X, L) \rightarrow\left(X^{\prime}, L^{\prime}\right)$ and $\left.f\right|_{L}: L \rightarrow L^{\prime}$ the restrictions of $f$.

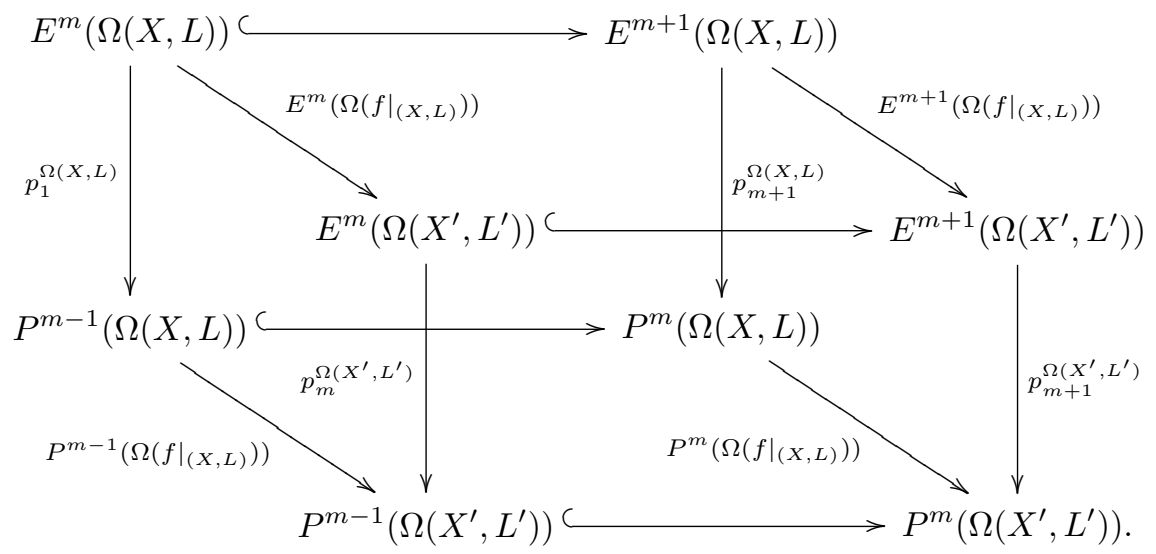

We give here another kind of naturality of the ladder (2.3) in $\mathcal{T}^{A}$ induced from the structure map $\sigma: K \rightarrow P^{m}(\Omega(X, L))$ of $\operatorname{cat}(X ; K, L: A) \leq m$.

Lemma 2.2. For any triad $(X ; K, L: A)$ in $\mathcal{T}^{A}$ with a compression $\sigma: K \rightarrow P^{m}(\Omega(X, L))$ relative $A$ of the inclusion $K \hookrightarrow X$, there is a sequence of maps $\sigma_{n}: P^{n}(\Omega(X, K)) \rightarrow$ $P^{m+n}(\Omega(X, L))(n \geq 0)$ with $\sigma_{0}=\sigma$, which makes the following diagram commutative up to homotopy relative $A$.

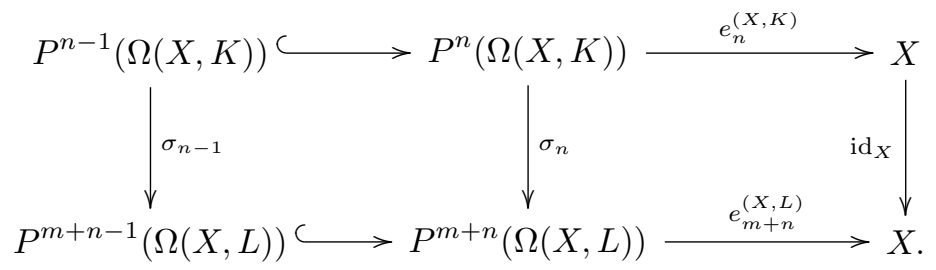

Proof. We construct $\sigma_{n}$ inductively on $n \geq 1$ : the homotopy commutativity relative $A$ of (2.5) without the dotted arrow induces a map of fibres in $\mathcal{T}, \hat{\sigma}_{n}: E^{n}(\Omega(X, K)) \rightarrow$ $E^{m+n}(\Omega(X, L))$. 


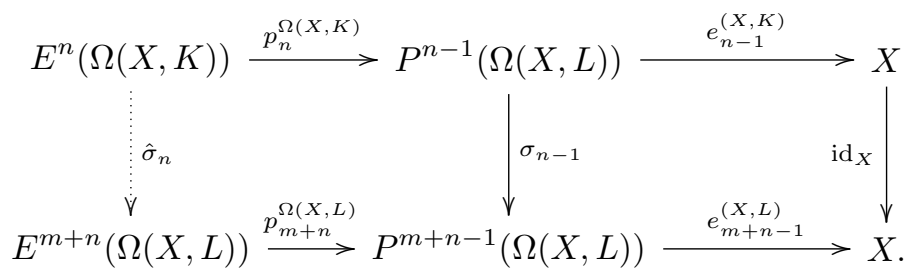

A standard argument shows that the homotopy commutativity of the left square implies the existence of $\sigma_{n}: P^{n}(\Omega(X, L)) \rightarrow P^{m+n}(\Omega(X, L))$ which makes (2.4) commutative up to homotopy relative $A$.

3. Properties of a new relative L-S category. Here we prove Theorem 1.14 and some consequences. For that we need

LEMma 3.1. $\operatorname{cat}(X ; K, L: A) \leq m$ if and only if the inclusion $g: K \hookrightarrow X$ is compressible into $P^{m}(\Omega(X, L)) \subset P^{\infty}(\Omega(X, L)) \simeq X$ relative $A$ as $\sigma: K \rightarrow P^{m}(\Omega(X, L))$ the structure map for $\operatorname{cat}(X ; K, L: A) \leq m$.

Proof. Let us assume that $\operatorname{cat}(X ; K, L: A) \leq m$. Then by the definition of the relative category, the diagonal map $\left.\Delta^{m+1}\right|_{K}: K \hookrightarrow X \rightarrow \prod^{m+1} X$ is compressible relative $A$ into $\mathrm{T}^{m+1}(X, L)$. This implies that there exists a map $\sigma$ from $K$ to $P^{m}(\Omega(X, L))$, which is a compression relative $A$ of the inclusion $g: K \hookrightarrow X$. Conversely, we assume that there is a compression relative $A$ of the inclusion $g: K \hookrightarrow X$ into $P^{m}(\Omega(X, L))$. Composing with $q_{m}: P^{m}(\Omega(X, L)) \rightarrow \mathrm{T}^{m+1}(X, L)$, we obtain a compression relative $A$ of the diagonal map $\left.\Delta^{m+1}\right|_{K}: K \hookrightarrow X \rightarrow \prod^{m+1} X$ into $\mathrm{T}^{m+1}(X, L)$. The following propositions complete the proof of Theorem 1.14 .

Proposition 3.2. Assume $X=K \supset L=A \supset *$. Then $\operatorname{cat}(X: A)=\operatorname{cat}(X ; X, A: A)=$ cat $^{\mathrm{FH}}(X, A)$.

Proof. By Lemma 3.1 with $X=K$ and $L=A$, $\operatorname{cat}(X ; X, A: A) \leq m$ if and only if there is a right homotopy inverse of $e_{m}^{(X ; X: A)}: P^{m}(\Omega(X: A)) \rightarrow X$ relative $A$, which is equivalent to cat $^{\mathrm{FH}}(X, K) \leq m$.

Proposition 3.3. Assume $X \supset K \supset L=A=*$. Then $\operatorname{cat}(X ; K)=\operatorname{cat}(X ; K, *: *)=$ cat $^{\mathrm{BG}}(X, K)$.

Proof. By Lemma 3.1 with $A=*$, $\operatorname{cat}(X ; K) \leq m$ if and only if the inclusion $K \hookrightarrow X$ is compressible into $P^{m}(\Omega(X))$, which is equivalent to cat ${ }^{\mathrm{BG}}(X, K) \leq m$.

Proposition 3.4. Assume $X=K \supset L \supset A=*$. Then $\operatorname{cat}(X, L)=\operatorname{cat}(X ; X, L: *)=$ cat $^{\mathrm{AL}}(X, L)$.

Proof. By Lemma 3.1 with $X=K$ and $A=*$, $\operatorname{cat}(X, L)=\operatorname{cat}(X ; X, L: *) \leq m$ if and only if there is a right homotopy inverse of $e_{m}^{(X ; X, L)}: P^{m}(\Omega(X, L)) \rightarrow X$, which is equivalent to cat $^{\mathrm{AL}}(X, L) \leq m$.

For relative L-S categories, one has: 
TheOrem 3.5. (1) Let $(X ; K, L: A)$ be a triad in $\mathcal{T}^{A}$. Then

$$
\begin{aligned}
& \operatorname{cat}(X ; K, L: A) \leq \operatorname{cat}(X ; K: A) \leq \operatorname{cat}(X ; L: A)+\operatorname{cat}(X ; K, L: A), \\
& \operatorname{cat}(X ; K, L: A) \leq \operatorname{cat}(X, L: A) \leq \operatorname{cat}(X, K: A)+\operatorname{cat}(X ; K, L: A) .
\end{aligned}
$$

More generally, for any maps $f:(L: A) \rightarrow(X: A)$ and $g:(K: A) \rightarrow(X: A)$,

$$
\begin{aligned}
& \operatorname{cat}(g, f) \leq \operatorname{cat}\left(g, *_{A}\right) \leq \operatorname{cat}\left(f, *_{A}\right)+\operatorname{cat}(g, f), \\
& \operatorname{cat}(g, f) \leq \operatorname{cat}\left(1_{(X: A)}, f\right) \leq \operatorname{cat}\left(1_{X}, g\right)+\operatorname{cat}(g, f),
\end{aligned}
$$

where $1_{X}:(X: A)=(X: A)$ denotes the identity and $*_{A}:(A: A) \hookrightarrow(X: A)$ denotes the trivial inclusion.

(2) If $\left(X^{\prime}, L^{\prime}: A\right) \supset(X, L: A)$ and $\left(K^{\prime}: A^{\prime}\right) \subset(K: A)$, then

$$
\begin{aligned}
\operatorname{cat}\left(X^{\prime} ; K^{\prime}, L^{\prime}: A^{\prime}\right) \leq \operatorname{Min}\left\{\operatorname{cat}\left(X^{\prime} ; K, L^{\prime}: A\right), \operatorname{cat}\left(X ; K^{\prime}, L: A^{\prime}\right)\right\} \\
\leq \operatorname{Max}\left\{\operatorname{cat}\left(X^{\prime} ; K, L^{\prime}: A\right), \operatorname{cat}\left(X ; K^{\prime}, L: A^{\prime}\right)\right\} \leq \operatorname{cat}(X ; K, L: A) .
\end{aligned}
$$

More generally, for any maps $f^{\prime}:\left(L^{\prime}: A\right) \rightarrow\left(X^{\prime}: A\right), f:(L: A) \rightarrow(X: A), g:$ $(K: A) \rightarrow(X: A), h:(X: A) \rightarrow\left(X^{\prime}: A\right), k:\left(K^{\prime}: A^{\prime}\right) \rightarrow(K: A)$ and $\ell:(L: A) \rightarrow\left(L^{\prime}: A\right)$ which satisfy the relation $f^{\prime} \circ \ell=h \circ f$, we have

$$
\begin{aligned}
& \operatorname{cat}\left(h \circ g \circ k, f^{\prime}\right) \leq \operatorname{Min}\left\{\operatorname{cat}\left(h \circ g, f^{\prime}\right), \operatorname{cat}(g \circ k, f)\right\} \\
& \leq \operatorname{Max}\left\{\operatorname{cat}\left(h \circ g, f^{\prime}\right), \operatorname{cat}(g \circ k, f)\right\} \leq \operatorname{cat}(g, f) .
\end{aligned}
$$

The following corollaries are immediate consequences of Theorem 3.5:

Corollary 3.6. (1) For a triad $(X ; K, L: *)$ in $\mathcal{T}_{*}$, we have

$$
\begin{aligned}
& \operatorname{cat}(X ; K, L) \leq \operatorname{cat}(X ; K)=\operatorname{cat}^{\mathrm{BG}}(X, K) \\
& \quad \leq \operatorname{cat}(X ; L)+\operatorname{cat}(X ; K, L)=\operatorname{cat}^{\mathrm{BG}}(X, L)+\operatorname{cat}(X ; K, L), \\
& \operatorname{cat}(X ; K, L) \leq \operatorname{cat}(X, L)=\operatorname{cat}^{\mathrm{AL}}(X, L) \\
& \quad \leq \operatorname{cat}(X, K)+\operatorname{cat}(X ; K, L)=\operatorname{cat}^{\mathrm{AL}}(X, K)+\operatorname{cat}(X ; K, L) .
\end{aligned}
$$

(2) For a pair $(X, L: A)$ in $\mathcal{T}^{A}$, we have

$$
\begin{aligned}
\operatorname{cat}(X, L: A) \leq \operatorname{cat}(X: A)=\operatorname{cat}^{\mathrm{FH}}(X, A) \\
\quad \leq \operatorname{cat}(X ; L: A)+\operatorname{cat}(X, L: A) \leq \operatorname{cat}(X ; L: A)+\operatorname{cat}^{\mathrm{FH}}(X, L) .
\end{aligned}
$$

If we further assume that $A=*$, then

$$
\operatorname{cat}(X, L) \leq \operatorname{cat}(X) \leq \operatorname{cat}(X ; L)+\operatorname{cat}(X, L) .
$$

(3) For maps $f: L \subset X, f^{\prime}: * \subset Y, g=1_{X}: X \rightarrow X, h: X \rightarrow Y, k=1_{X}: X \rightarrow X$ and $\ell: L \rightarrow *$ in $\mathcal{T}_{*}$ with $\left.h\right|_{L}=\ell$, we have

$$
\operatorname{cat}^{\mathrm{BG}}(h)=\operatorname{cat}(h, *)=\operatorname{cat}\left(h \circ g, f^{\prime}\right) \leq \operatorname{cat}(g, f)=\operatorname{cat}^{\mathrm{AL}}(X, L) .
$$

In Definition 1.6, we have $\operatorname{cat}\left(X ; F_{i}, F_{i-1}: A\right) \leq 1$ for the filtration $\left\{F_{i}\right\}$. Hence we have:

Corollary 3.7. $\operatorname{cat}\left(X ; F_{i}, A: A\right) \leq i$ for every $i$. 
Proof of Theorem 3.5. The case of maps is left to the reader, and we concentrate on the case of spaces.

Firstly, we show (1) for a triad $(X ; K, L: A)$ in $\mathcal{T}^{A}$ :

To show $\operatorname{cat}(X ; K, L: A) \leq \operatorname{cat}(X ; K: A)$, we assume that $\operatorname{cat}(X ; K: A)=m$. By Lemma 3.1 for the triad $(X ; K, A: A), \operatorname{cat}(X ; K: A)=\operatorname{cat}(X ; K, A: A) \leq m$ if and only if there is a compression $\sigma: K \rightarrow P^{m}(\Omega(X: A))$ relative $A$ of the inclusion $K \hookrightarrow X$. By Lemma 2.1 for the inclusion $(X ; K, A: A) \hookrightarrow(X ; K, L: A)$, the composition $P^{m}\left(\Omega\left(\left.f\right|_{X: A}\right)\right) \circ \sigma: K \rightarrow$ $P^{m}(\Omega(X, L))$ gives a compression of the inclusion $K \hookrightarrow X$, which implies $\operatorname{cat}(X ; K, L: A)$ $\leq m=\operatorname{cat}(X ; K: A)$.

To show $\operatorname{cat}(X ; K, L: A) \leq \operatorname{cat}(X, L: A)$, we assume that $\operatorname{cat}(X, L: A)=m$. By Lemma 3.1 for the triad $(X ; X, L: A)$, $\operatorname{cat}(X, L: A) \leq m$ if and only if there is a compression $\sigma: X \rightarrow P^{m}(\Omega(X, L))$ relative $A$ of the identity $1_{X}$. By restricting $\sigma$ to $K$, we obtain a compression $\left.\sigma\right|_{K}: K \rightarrow P^{m}(\Omega(X, L))$ relative $A$ of the inclusion $K \hookrightarrow X$, which implies $\operatorname{cat}(X ; K, L: A) \leq m=\operatorname{cat}(X . L: A)$.

To show the inequality $\operatorname{cat}(X ; K: A) \leq \operatorname{cat}(X ; L: A)+\operatorname{cat}(X ; K, L: A)$, we assume that $\operatorname{cat}(X ; L: A)=m$ and $\operatorname{cat}(X ; K, L: A)=n$. By Lemma 3.1 for the $\operatorname{triad}(X ; L, A: A)$, $\operatorname{cat}(X ; L: A) \leq m$ if and only if there is a compression $\sigma: L \rightarrow P^{m}(\Omega(X: A))$ relative $A$ of the inclusion $L \hookrightarrow X$. Then by Lemma 2.2 for the triad $(X ; L, A: A)$, we have the following commutative ladder with $\sigma_{0}=\sigma$ up to homotopy relative $A$ :

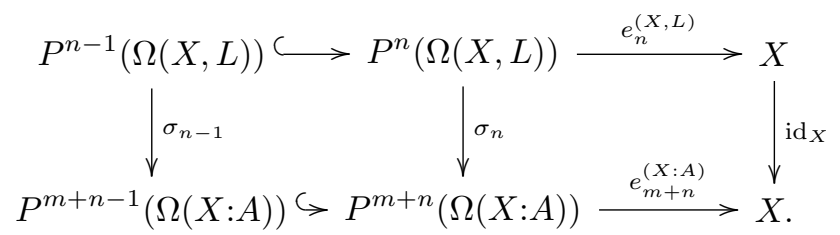

Again by Lemma 3.1 for the $\operatorname{triad}(X ; K, L: L)$, cat $(X ; K, L: A) \leq n$ if and only if there is a compression $\tau: K \rightarrow P^{n}(\Omega(X, L))$ relative $A$ of the inclusion $K \hookrightarrow X$. Then the composition $\sigma_{n} \circ \tau: K \rightarrow P^{m+n}(\Omega(X: A))$ gives a compression relative $A$ of the inclusion $K \hookrightarrow X$, which implies that $\operatorname{cat}(X ; K: A) \leq m+n=\operatorname{cat}(X ; L: A)+\operatorname{cat}(X ; K, L: A)$.

To show the inequality $\operatorname{cat}(X, L: A) \leq \operatorname{cat}(X, K: A)+\operatorname{cat}(X ; K, L: A)$, we assume that $\operatorname{cat}(X ; K, L: A)=m$ and $\operatorname{cat}(X, K: A)=n$. By Lemma 3.1 for the $\operatorname{triad}(X ; K, L: A)$, $\operatorname{cat}(X ; K, L: A) \leq m$ if and only if there is a compression $\tau: K \rightarrow P^{m}(\Omega(X, L))$ relative $A$ of the inclusion $K \hookrightarrow X$. Then by Lemma 2.2 for the triad $(X ; K, L: A)$, we have the following commutative ladder with $\tau_{0}=\tau$ up to homotopy relative $A$ :

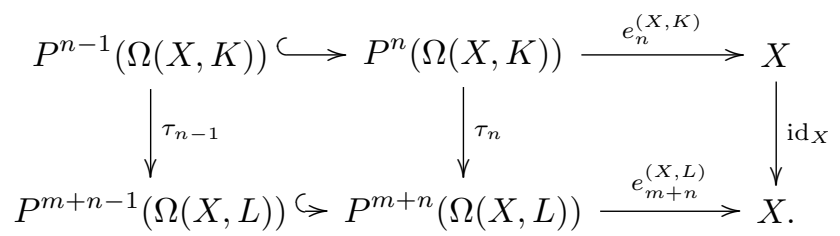

Again by Lemma 3.1 for the triad $(X ; X, K: A)$, cat $(X, K: A) \leq n$ if and only if there is a compression $\rho: X \rightarrow P^{n}(\Omega(X, K))$ relative $A$ of the identity $1_{X}: X \rightarrow X$. Then the composition $\tau_{n} \circ \rho: X \rightarrow P^{m+n}(\Omega(X, L))$ gives a compression relative $A$ of the identity $1_{X}: X \rightarrow X$, which implies that $\operatorname{cat}(X, L: A) \leq m+n=\operatorname{cat}(X, K: A)+\operatorname{cat}(X ; K, L: A)$. 
Secondly, we show $(2)$ for a triad $(X ; K, L: A)$ with spaces $X^{\prime} \supset X,\left(K^{\prime}: A^{\prime}\right) \subset(K: A)$ and $\left(L^{\prime}: A^{\prime}\right) \subset(L: A)$, which is sufficient to show that $\operatorname{cat}\left(X^{\prime} ; K, L^{\prime}: A\right) \leq \operatorname{cat}(X ; K, L: A)$ and $\operatorname{cat}\left(X ; K^{\prime}, L: A^{\prime}\right) \leq \operatorname{cat}(X ; K, L: A)$ :

To show $\operatorname{cat}\left(X^{\prime} ; K, L^{\prime}: A\right) \leq \operatorname{cat}(X ; K, L: A)$, we assume that $\operatorname{cat}(X ; K, L: A)=m$. By Lemma 3.1 for the triad $(X ; K, L: A), \operatorname{cat}(X ; K, L: A) \leq m$ if and only if there is a compression $\sigma: K \rightarrow P^{m}(\Omega(X, L))$ relative $A$ of the inclusion $K \hookrightarrow X$. Since $X^{\prime} \supset X$, we have the inclusion of triads : $(X ; K, L: A) \hookrightarrow\left(X^{\prime} ; K, L^{\prime}: A\right)$. Then by Lemma 2.1 for the map of triads $j:(X ; K, L: A) \hookrightarrow\left(X^{\prime} ; K, L^{\prime}: A\right)$, we have the following commutative ladder up to homotopy relative $A$ :

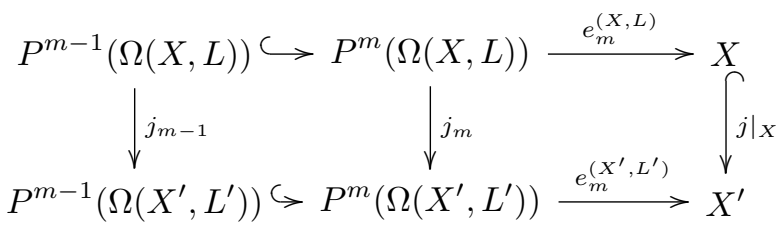

with $j_{0}=\operatorname{id}_{L}$ and $j_{k}=P^{k}\left(\Omega\left(\left.j\right|_{(X, L)}\right)\right), 1 \leq k \leq m$. Thus the map $j_{m} \circ \sigma$ gives a compression relative $A$ of the inclusion $K \hookrightarrow X \subset X^{\prime}$, and hence $\operatorname{cat}\left(X^{\prime} ; K, L^{\prime}: A\right) \leq m$ $=\operatorname{cat}(X ; K, L: A)$.

To show $\operatorname{cat}\left(X ; K^{\prime}, L: A^{\prime}\right) \leq \operatorname{cat}(X ; K, L: A)$, we may assume that $A=A^{\prime}$, since it is clear by definition that $\operatorname{cat}\left(X ; K, L: A^{\prime}\right) \leq \operatorname{cat}(X ; K, L: A)$ if $A^{\prime} \subset A$ : let us assume that $\operatorname{cat}(X ; K, L: A)=m$. By Lemma 3.1 for the $\operatorname{triad}(X ; K, L: A), \operatorname{cat}(X ; K, L: A) \leq m$ if and only if there is a compression $\sigma: K \rightarrow P^{m}(\Omega(X, L))$ relative $A$ of the inclusion $K \hookrightarrow X$. Hence the restriction $\left.\sigma\right|_{K^{\prime}}$ of the map $\sigma$ to $K^{\prime}$ gives a compression relative $A$ of the inclusion $K^{\prime} \hookrightarrow X$, and hence $\operatorname{cat}\left(X ; K^{\prime}, L: A\right) \leq m=\operatorname{cat}(X ; K, L: A)$.

4. A higher Hopf invariant for a triad. Let us consider the following exact sequences of abelian groups and algebraic loops:

$$
\begin{aligned}
& 0 \rightarrow\left[\Sigma V, E^{m+1}(\Omega(X, L))\right] \stackrel{p_{m+1 *}^{(X, L)}}{\longrightarrow}\left[\Sigma V, P^{m}(\Omega(X, L))\right] \stackrel{e_{m *}^{(X, L)}}{\longrightarrow}[\Sigma V, X] \rightarrow 0, \\
& 1 \rightarrow\left[V, E^{m+1}(\Omega(X, L))\right] \stackrel{p_{m+1 *}^{(X, L)}}{\longrightarrow}\left[V, P^{m}(\Omega(X, L))\right] \stackrel{e_{m *}^{(X)}}{\longrightarrow}[V, X] .
\end{aligned}
$$

Since the fibre $\Omega(X)$ of a fibration $p_{m+1}^{(X, L)}$ is contractible in the total space $E^{m+1}(\Omega(X, L))$ of $p_{m+1}^{(X, L)}$, we know $e_{m *}^{(X, L)}:\left[\Sigma V, P^{m}(\Omega(X, L))\right] \rightarrow[\Sigma V, X]$ is an epimorphism of abelian groups and $p_{m+1 *}^{(X, L)}:\left[\Sigma V, E^{m+1}(\Omega(X, L))\right] \rightarrow\left[\Sigma V, P^{m}(\Omega(X, L))\right]$ is a monomorphism of abelian groups. Similarly, $p_{m+1 *}^{(X, L)}:\left[V, E^{m+1}(\Omega(X, L))\right] \rightarrow\left[V, P^{m}(\Omega(X, L))\right]$ is a monomorphism of algebraic loops. Thus we obtain the following proposition:

Proposition 4.1. (1) $e_{m *}^{(X, L)}:\left[\Sigma V, P^{m}(\Omega(X, L))\right] \rightarrow[\Sigma V, X]$ is an epimorphism of abelian groups.

(2) $p_{m+1 *}^{(X, L)}:\left[\Sigma V, E^{m+1}(\Omega(X, L))\right] \rightarrow\left[\Sigma V, P^{m}(\Omega(X, L))\right]$ is a monomorphism of abelian groups.

(3) $p_{m+1 *}^{(X, L)}:\left[V, E^{m+1}(\Omega(X, L))\right] \rightarrow\left[V, P^{m}(\Omega(X, L))\right]$ is a monomorphism of algebraic loops. 
We give here a definition of higher Hopf invariants in a slightly different form: let $(X ; K, L: A)$ be a triad in $\mathcal{T}^{A}$, let $V$ be a co-loop co-H-space, and let $\alpha: V \rightarrow K$ be a map in $\mathcal{T}$ such that $X \supset \hat{K}=K \cup_{\alpha} C V \supset K$. We assume that $\operatorname{cat}(X ; K, L: A) \leq m$. Then by Lemma 3.1 for the triad $(X ; K, L: A)$, $\operatorname{cat}(X ; K, L: A) \leq m$ implies that the inclusion $i: K \hookrightarrow X$ is compressible into $P^{m}(\Omega(X, L))$ relative $A$ as a map $\sigma: K \rightarrow P^{m}(\Omega(X, L))$. Since $e_{m}^{(X, L)} \circ \sigma \circ \alpha \sim i \circ \alpha$ is trivial in $\hat{K} \subset X$, we obtain a unique lift $H_{m}^{\sigma}(\alpha): V \rightarrow$ $E^{m+1}(\Omega(X, L)) \simeq \Omega(X, L) * \Omega(X) * \cdots * \Omega(X)$ of $\sigma \circ \alpha$.

Definition 4.2. We define $H_{m}^{(X ; K, L: A)}(\alpha)$ as follows:

$$
\begin{aligned}
H_{m}^{(X ; K, L: A)}(\alpha) & =\left\{\left[H_{m}^{\sigma}(\alpha)\right] \mid \begin{array}{l}
\sigma: K \rightarrow P^{m}(\Omega(X, L)) \text { is a compression rela- } \\
\text { tive } A \text { of the inclusion } K \hookrightarrow X
\end{array}\right. \\
& \subset[V, \Omega(X, L) * \Omega(X) * \cdots * \cdots(X)] .
\end{aligned}
$$

Now let $(K, L: A)$ be a pair in $\mathcal{T}^{A}$ and let $\alpha: V \rightarrow K$ a map in $\mathcal{T}$. We assume that $\operatorname{cat}(K, L: A) \leq m$. By Lemma 3.1 for the triad $(K ; K, L: A), \operatorname{cat}(K, L: A) \leq m$ implies that the identity $1_{K}: K \rightarrow K$ is compressible into $P^{m}(\Omega(K, L))$ relative $A$ as a map $\sigma: K \rightarrow P^{m}(\Omega(K, L))$. By Lemma 2.1 for the inclusion $j:(K ; K, *: *) \hookrightarrow(K ; K, L: *)$, the following ladder is commutative up to homotopy:

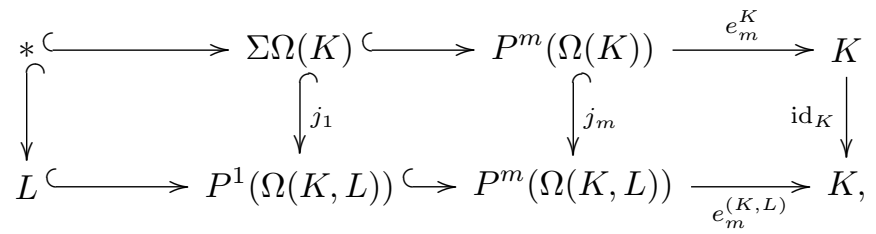

where $e_{1}^{K}=\left.e_{m}^{K}\right|_{\Sigma \Omega(K)}: \Sigma \Omega(K) \rightarrow K$ is given by the evaluation map (see Ganea [9] or [14]). Since $V$ is a co-loop co-H-space, the evaluation map $e_{1}^{V}: \Sigma \Omega(V) \rightarrow V$ admits a right homotopy inverse, say the co-H-structure map $\rho^{V}: V \rightarrow \Sigma \Omega(V)$ for $V$, by Ganea [10]. Then we have $e_{1}^{K} \circ \Sigma \Omega(\alpha) \circ \rho^{V} \sim \alpha \circ e_{1}^{V} \circ \rho^{V} \sim \alpha$, and hence $e_{1}^{(K, L)} \circ j_{1} \circ \Sigma \Omega(\alpha) \circ \rho^{V} \simeq$ $\operatorname{id}_{K \circ} e_{1}^{K} \circ \Sigma \Omega(\alpha) \circ \rho^{V} \sim \alpha$. Since both maps $e_{1}^{(K, L)} \circ \sigma \circ \alpha$ and $e_{1}^{(K, L)} \circ j_{1} \circ \Sigma \Omega(\alpha) \circ \rho^{V}$ are homotopic to $\alpha$, the difference $d(\alpha)=\sigma \circ \alpha-j_{1} \circ \Sigma \Omega(\alpha) \circ \rho^{V}$ is trivial in $K$. Thus we obtain a unique lift $H_{m}^{\sigma}(\alpha): V \rightarrow E^{m+1}(\Omega(K, L)) \simeq \Omega(X, L) * \Omega(X) * \cdots * \cdots \cdot \Omega(X)$ of $d(\alpha)$.

Definition 4.3. We define $H_{m}^{(K, L: A)}(\alpha)$ as follows:

$$
\begin{aligned}
H_{m}^{(K, L: A)}(\alpha) & =\left\{\left[H_{m}^{\sigma}(\alpha)\right] \mid \sigma \text { is a compression relative } A \text { of } 1_{K}\right\} \\
& \subset[V, \Omega(K, L) * \Omega(K) * \cdots * \cdots(K)] .
\end{aligned}
$$

Proof of Theorem 1.15. Let $(X ; K, L: A)$ be a triad in $\mathcal{T}^{A}, V$ be a co-loop co-H-space and $\alpha: V \rightarrow K$ be a map in $\mathcal{T}$ such that $X \supset \hat{K}=K \cup_{\alpha} C V \supset K$. Assuming $\operatorname{cat}(X ; K, L: A) \leq m$ and $H_{m}^{(X ; K, L: A)}(\alpha)=0$, we show $\operatorname{cat}(X ; \hat{K}, L: A) \leq m$ : by the assumption, there is a compression $\sigma: K \rightarrow P^{m}(\Omega(X, L))$ relative $A$ of the inclusion $K \hookrightarrow$ $X$ such that $\sigma \circ \alpha \sim p_{m+1}^{(X, L)} \circ H_{m}^{\sigma}(\alpha) \sim *$, and hence there is a map $\hat{\sigma}: \hat{K} \rightarrow P^{m}(\Omega(X, L))$ whose restriction to $K$ is $\sigma$. Since $e_{m}^{(X, L)} \circ \sigma$ and the inclusion $K \hookrightarrow X$ are homotopic relative $A$, the difference between $e_{m}^{(X, L)} \circ \hat{\sigma}$ and the inclusion $\hat{K} \hookrightarrow X$ is given by an element $[\delta] \in[\Sigma V, X]$. By Proposition $4.1(1)$, we have a map $\hat{\delta}: \Sigma V \rightarrow P^{m}(\Omega(X, L))$ such that $e_{m}^{(X, L)} \circ \hat{\delta} \sim \delta$. By subtracting $\hat{\delta}$ from $\hat{\sigma}$, we obtain a genuine compression 
$\sigma^{\prime}=\hat{\sigma}-\hat{\delta}: \Sigma V \rightarrow P^{m}(\Omega(X, L))$ of the inclusion $\hat{K} \rightarrow P^{m}(\Omega(X, L))$ relative $A$, where the subtraction is given by the co-action of $\Sigma V$ under $K \cup_{\alpha} C^{2} V=\hat{K}$ the mapping cone of $\alpha$. This implies that $\operatorname{cat}(X ; \hat{K}, L: A) \leq m$.

We describe here the relationship among higher Hopf invariants. The following definition is essentially due to Berstein and Hilton [3]:

Definition 4.4. Let $(X ; K, L: A)$ and $\left(X^{\prime} ; K^{\prime}, L^{\prime}: A\right)$ be triads in $\mathcal{T}^{A}, V$ be a co-loop co-H-space, and $s: K \rightarrow \mathrm{T}^{m+1}(X, L)$ and $s^{\prime}: K^{\prime} \rightarrow \mathrm{T}^{m+1}\left(X^{\prime}, L^{\prime}\right)$ be compressions of $\Delta^{m+1} \circ i: K \hookrightarrow \prod^{m+1} X$ and $\Delta^{m+1} \circ i^{\prime}: K^{\prime} \hookrightarrow \prod^{m+1} X^{\prime}$ relative $A$, respectively, so that $\operatorname{cat}(X ; K, L: A) \leq m$ and $\operatorname{cat}\left(X^{\prime} ; K^{\prime}, L^{\prime}: A\right) \leq m$. A map $f:(X ; K, L: A) \rightarrow$ $\left(X^{\prime} ; K^{\prime}, L^{\prime}: A\right.$ ) of triads in $\mathcal{T}^{A}$ is called $m$-primitive (with respect to $s$ and $s^{\prime}$ ), if $\left.s^{\prime} \circ f\right|_{K} \sim$ $\mathrm{T}^{m+1}\left(\left.f\right|_{\left(X^{\prime}, L^{\prime}\right)}\right) \circ s$.

Let $(X ; K, L: A)$ and $\left(X^{\prime} ; K^{\prime}, L^{\prime}: A\right)$ be triads in $\mathcal{T}^{A}$, and let $\operatorname{cat}(X ; K, L: A) \leq m$ and $\operatorname{cat}\left(X^{\prime} ; K^{\prime}, L^{\prime}: A\right) \leq m$ with compressions $s: K \rightarrow \mathrm{T}^{m+1}(X, L)$ and $s^{\prime}: K^{\prime} \rightarrow$ $\mathrm{T}^{m+1}\left(X^{\prime}, L^{\prime}\right)$ of $\Delta^{m+1} \circ i: K \hookrightarrow \prod^{m+1} X$ and $\Delta^{m+1} \circ i^{\prime}: K^{\prime} \hookrightarrow \prod^{m+1} X^{\prime}$ relative $A$, respectively. By using the lower right square of the diagram (2.2), we obtain structure maps $\sigma, \sigma^{\prime}$ for $\operatorname{cat}(X ; K, L: A) \leq m$ and $\operatorname{cat}\left(X^{\prime} ; K^{\prime}, L^{\prime}: A\right) \leq m$ corresponding to $s$ and $s^{\prime}$, respectively by $s \sim q_{m}^{(X, L)} \circ \sigma$ and $s^{\prime} \sim q_{m}^{\left(X^{\prime}, L^{\prime}\right)} \circ \sigma^{\prime}$ relative $A$.

Lemma 4.5. Let $f:(X ; K, L: A) \rightarrow\left(X^{\prime} ; K^{\prime}, L^{\prime}: A\right)$ be a map of triads in $\mathcal{T}^{A}$. Then $f$ is $m$-primitive with respect to $s$ and $s^{\prime}$, if and only if $\left.\sigma^{\prime} \circ f\right|_{K} \sim P^{m}\left(\Omega\left(\left.f\right|_{(X, L)}\right)\right) \circ \sigma$ relative $A$ for the corresponding structure maps $\sigma$ and $\sigma^{\prime}$.

Proof. Assume that $f$ satisfies that $\left.\sigma^{\prime} \circ f\right|_{K} \sim P^{m}\left(\Omega\left(\left.f\right|_{(X, L)}\right)\right) \circ \sigma$. By composing $q_{m}^{\left(X^{\prime}, L^{\prime}\right)}$ : $P^{m}\left(\Omega\left(X^{\prime}, L^{\prime}\right)\right) \rightarrow \mathrm{T}^{m+1}\left(X^{\prime}, L^{\prime}\right)$ with both sides, we obtain

$$
\begin{aligned}
\left.s^{\prime} \circ f\right|_{K} & \left.\sim q_{m}^{\left(X^{\prime}, L^{\prime}\right)} \circ \sigma^{\prime} \circ f\right|_{K} \sim q_{m}^{\left(X^{\prime}, L^{\prime}\right)} \circ P^{m}\left(\Omega\left(\left.f\right|_{(X, L)}\right)\right) \circ \sigma \\
& \sim \mathrm{T}^{m+1}\left(\left.f\right|_{(X, L)}\right) \circ q_{m}^{(X, L)} \circ \sigma \sim \mathrm{T}^{m+1}\left(\left.f\right|_{(X, L)}\right) \circ s
\end{aligned}
$$

relative $A$, and hence $f$ is $m$-primitive with respect to $s$ and $s^{\prime}$. Conversely assume that $f$ is $m$-primitive with respect to $s$ and $s^{\prime}$. Then the naturality of the lower right square of the diagram (2.2) immediately induces the homotopy $\left.\sigma^{\prime} \circ f\right|_{K} \sim P^{m}\left(\Omega\left(\left.f\right|_{(X, L)}\right)\right) \circ \sigma$ relative $A$.

Theorem 4.6. Let $(X ; K, L: A)$ and $\left(X^{\prime} ; K^{\prime}, L^{\prime}: A\right)$ be triads in $\mathcal{T}^{A}$, $V$ be a co-loop co$H$-space, and $s: K \rightarrow \mathrm{T}^{m+1}(X, L)$ and $s^{\prime}: K^{\prime} \rightarrow \mathrm{T}^{m+1}\left(X^{\prime}, L^{\prime}\right)$ be compressions of the inclusions $i: K \hookrightarrow X$ and $i^{\prime}: K^{\prime} \hookrightarrow X^{\prime}$ relative $A$, respectively, so that $\operatorname{cat}(X ; K, L: A) \leq$ $m$ and $\operatorname{cat}\left(X^{\prime} ; K^{\prime}, L^{\prime}: A\right) \leq m$, respectively. Let $f:(X ; K, L: A) \rightarrow\left(X^{\prime} ; K^{\prime}, L^{\prime}: A\right)$ be a map of triads in $\mathcal{T}^{A}$ and let $\alpha: V \rightarrow K$ be a map in $\mathcal{T}$ such that $X \supset \hat{K}=K \cup_{\alpha} C V \supset K$ and $X^{\prime} \supset \hat{K}^{\prime}=K^{\prime} \cup_{\left.f\right|_{K} \circ \alpha} C V \supset K$. If $f$ is $m$-primitive with respect to $s$ and $s^{\prime}$, then

$$
E^{m+1}\left(\Omega\left(\left.f\right|_{(X, L)}\right)\right)_{\#} \circ H_{m}^{(X ; K, L: A)}(\alpha) \subset H_{m}^{\left(X^{\prime} ; K^{\prime}, L^{\prime}: A\right)}\left(\left.f\right|_{K} \circ \alpha\right) .
$$

Proof. By Lemma 2.1 for $f:(X ; K, L: A) \rightarrow\left(X^{\prime} ; K^{\prime}, L^{\prime}: A\right)$ a map of triads in $\mathcal{T}^{A}$, the following diagram is commutative up to homotopy relative $A$ : 


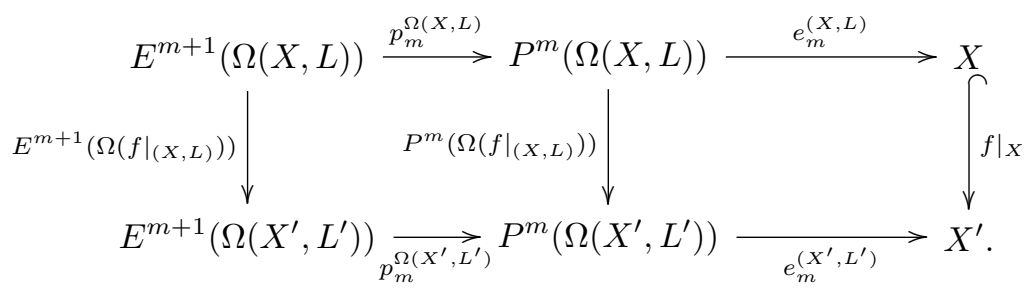

Since $f$ is $m$-primitive with respect to $s$ and $s^{\prime}$, we have the homotopy relation relative $\left.A P^{m}\left(\Omega\left(\left.f\right|_{(X, L)}\right)\right) \circ \sigma \sim \sigma^{\prime} \circ f\right|_{K}$ for the corresponding compressions $\sigma$ and $\sigma^{\prime}$ relative $A$ of the inclusions $i: K \hookrightarrow X$ and $i^{\prime}: K^{\prime} \hookrightarrow X^{\prime}$, resp. Thus we have the following homotopy relation:

$$
\begin{aligned}
& p_{m}^{\Omega\left(X^{\prime}, L^{\prime}\right)} \circ E^{m+1}\left(\Omega\left(\left.f\right|_{(X, L)}\right)\right) \circ H_{m}^{\sigma}(\alpha) \\
& \sim P^{m}\left(\Omega\left(\left.f\right|_{(X, L)}\right)\right) \circ p_{m}^{\Omega(X, L)} \circ H_{m}^{(X ; K, L: A)}(\alpha) \\
&\left.\sim P^{m}\left(\Omega\left(\left.f\right|_{(X, L)}\right)\right) \circ \sigma \circ \alpha \sim \sigma^{\prime} \circ f\right|_{K} \circ \alpha \sim p_{m}^{\Omega\left(X^{\prime}, L^{\prime}\right)} \circ H_{m}^{\sigma^{\prime}}\left(\left.f\right|_{K} \circ \alpha\right) .
\end{aligned}
$$

Hence we obtain $E^{m+1}\left(\Omega\left(\left.f\right|_{(X, L)}\right) \circ H_{m}^{\sigma}(\alpha) \sim H_{m}^{\sigma^{\prime}}\left(\left.f\right|_{K} \circ \alpha\right)\right.$, since $p_{m *}^{\Omega\left(X^{\prime}, L^{\prime}\right)}$ is monic by Proposition 4.1 (3). Thus we have

$$
E^{m+1}\left(\Omega\left(\left.f\right|_{(X, L)}\right)\right)_{\#} H_{m}^{(X ; K, L: A)}(\alpha) \subset H_{m}^{\left(X^{\prime} ; K^{\prime}, L^{\prime}: A\right)}\left(\left.f\right|_{K} \circ \alpha\right) .
$$

This completes the proof of Theorem 4.6.

TheOREM 4.7. Let $(X, K, L: A)$ be a triple in $\mathcal{T}^{A}, V$ be a co-loop co-H-space, and $\alpha$ : $V \rightarrow K$ be a map in $\mathcal{T}$ such that $X \supset \hat{K}=K \cup_{\alpha} C V \supset K$. If cat $(K, L: A) \leq m$, then

$$
E^{m+1}\left(\Omega\left(\left.j\right|_{(K, L)}\right)\right)_{\#} \circ H_{m}^{(K, L: A)}(\alpha) \subset H_{m}^{(X ; K, L: A)}(\alpha),
$$

where $j:(K ; K, L: A) \rightarrow(X ; K, L: A)$ is the inclusion.

Corollary 4.8. For the filtration $\left\{F_{i}\right\}$ in Definition 1.6, we have

$$
E^{m+1}\left(\Omega\left(\left.j_{i}\right|_{\left(F_{i}, F_{i-1}\right)}\right)\right)_{\#} \circ H_{i}^{\left(F_{i}, F_{i-1}: A\right)}(\alpha) \subset H_{i}^{\left(X ; F_{i}, F_{i-1}: A\right)}(\alpha)
$$

for every $i$, where $j_{i}:\left(F_{i} ; F_{i}, F_{i-1}: A\right) \hookrightarrow\left(X ; F_{i}, F_{i-1}: A\right)$ denote the inclusion.

Proof. Proof of Theorem 4.7 Let $(X, K, L: A)$ be a triple in $\mathcal{T}^{A}, V$ be a co-loop co-Hspace and $\alpha: V \rightarrow K$ be a map in $\mathcal{T}$ such that $X \supset \hat{K}=K \cup_{\alpha} C V \supset K$. Assuming $\operatorname{cat}(K, L: A) \leq m$, we show $E^{m+1}\left(\Omega\left(\left.j\right|_{(K, L)}\right)\right)_{\#} H_{m}^{(K, L: A)}(\alpha) \subset H_{m}^{(X ; K, L: A)}(\alpha)$, where $j$ : $(K ; K, L: A) \rightarrow(X ; K, L: A)$ denotes the inclusion: By Lemma 2.1 for $j:(K ; K, L: A) \rightarrow$ $(X ; K, L: A)$ an inclusion map of triads in $\mathcal{T}^{A}$, the following diagram is commutative up to homotopy relative $A$ :

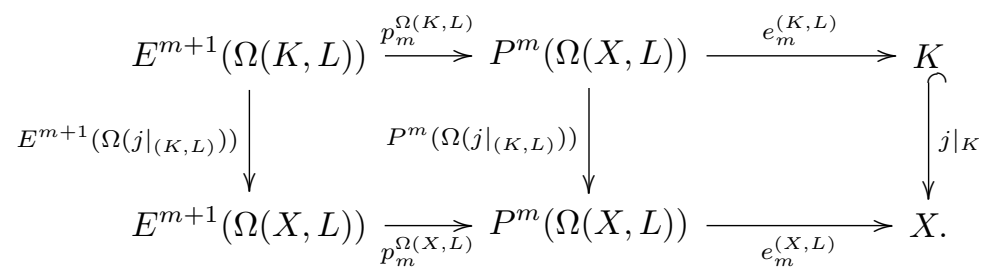

From the definition of a higher Hopf invariant, we obtain $p_{m}^{\Omega(K, L)} \circ H_{m}^{\sigma}(\alpha) \sim \sigma \circ \alpha-$ 
$j_{1} \circ \Sigma \Omega(\alpha) \circ \rho^{V}$, and hence we have the homotopy relation

$$
\begin{aligned}
& p_{m}^{\Omega(X, L)} \circ E^{m+1}\left(\Omega\left(\left.j\right|_{(K, L)}\right)\right) \circ H_{m}^{\sigma}(\alpha) \sim P^{m}\left(\Omega\left(\left.j\right|_{(K, L)}\right)\right) \circ p_{m}^{\Omega(K, L)} \circ H_{m}^{\sigma}(\alpha) \\
& \sim P^{m}\left(\Omega\left(\left.j\right|_{(K, L)}\right)\right) \circ \sigma \circ \alpha-P^{m}\left(\Omega\left(\left.j\right|_{(K, L)}\right)\right) \circ j_{1} \circ \Sigma \Omega(\alpha) \circ \rho^{V} \\
& \sim P^{m}\left(\Omega\left(\left.j\right|_{(K, L)}\right)\right) \circ \sigma \circ \alpha-j_{1} \circ \Sigma \Omega\left(\left.j\right|_{(K, L)}\right) \Sigma \Omega(\alpha) \circ \rho^{V} \\
& \sim P^{m}\left(\Omega\left(\left.j\right|_{(K, L)}\right)\right) \circ \sigma \circ \alpha-j_{1} \circ \Sigma \Omega\left(\left.j\right|_{(K, L)} \circ \alpha\right) \circ \rho^{V} \\
& \sim P^{m}\left(\Omega\left(\left.j\right|_{(K, L)}\right)\right) \circ \sigma \circ \alpha,
\end{aligned}
$$

since $\left.j\right|_{(K, L)} \circ \alpha \sim *$ in $X$. This implies that $E^{m+1}\left(\Omega\left(\left.j\right|_{(K, L)}\right)\right) \circ H_{m}^{\sigma}(\alpha)$ is homotopic to $H_{m}^{P^{m}\left(\Omega\left(\left.j\right|_{(K, L)}\right)\right) \circ \sigma}(\alpha)$, and hence $E^{m+1}\left(\Omega\left(\left.j\right|_{(K, L)}\right)\right)_{\#} \circ H_{m}^{(K, L: A)}(\alpha) \subset H_{m}^{(X ; K, L: A)}(\alpha)$.

5. Categorical length. Let $F_{i}^{X}, 0 \leq i \leq m$, and $F_{j}^{Y}, 0 \leq j \leq n$, be categorical sequences for $(X: A) \in \mathcal{T}^{A}$ and $(Y: A) \in \mathcal{T}^{A}$, respectively. Then for a map $f:(X: A) \rightarrow$ $(Y: A)$, we say that $f$ preserves categorical sequences, if $f\left(F_{i}^{X}\right) \subset F_{i}^{Y}$ for all $i \geq 0$. We first show the following:

Lemma 5.1. Let $(X: A) \in \mathcal{T}^{A}$ be dominated by $(Y: A) \in \mathcal{T}^{A}$ with a categorical sequence of length $m$. Then there is a categorical sequence for $(X: A)$ of length $m$ compatible with the given categorical sequence for $(Y: A)$, i.e., the inclusion $i:(X: A) \hookrightarrow(Y: A)$ and the retraction $r:(Y: A) \rightarrow(X: A)$ preserve categorical sequences.

The above lemma implies the relationship between the L-S category and the categorical length.

Proof of Theorem 1.16. Assume catlen $(X: A)=m$ with a categorical sequence $\left(F_{i}^{X}: A\right)$, $0 \leq i \leq m$ for $(X: A)$. Then by Corollary 3.7 , we have $\operatorname{cat}(X: A)=\operatorname{cat}(X ; X: A)=$ $\operatorname{cat}\left(X ; F_{m}^{X}: A\right) \leq m=\operatorname{catlen}(X: A)$. Hence we have $\operatorname{cat}(X: A) \leq \operatorname{catlen}(X: A)$. Conversely assume $\operatorname{cat}(X: A)=m$. Then the pair $(X: A)$ is dominated by $\left(P^{m}(\Omega(X: A)): A\right)$ which has the cone decomposition $\left(P^{i}(\Omega(X: A)): A\right), 0 \leq i \leq m$ as the canonical categorical sequence. Thus by Lemma 5.1 , we have that $(X: A)$ has also a categorical sequence of length $m$, and hence that catlen $(X: A) \leq m=\operatorname{cat}(X: A)$. This completes the proof of Theorem 1.16.

Proof of Lemma 5.1 Let $\left(F_{i}^{Y}: A\right), 0 \leq i \leq m$, be a categorical sequence for $(Y: A) \in \mathcal{T}^{A}$ and $\sigma: X \rightarrow Y$ and $\rho: Y \rightarrow X$ be maps such that $\rho \circ \sigma \sim 1_{X}$. Then we define $F_{i}$ as the homotopy pullback of $\sigma$ and the inclusion $\iota_{i}: F_{i}^{Y} \hookrightarrow F_{m}^{Y}$. Since the image of $\left.\sigma\right|_{A}$ is the same as the inclusion $A \subseteq F_{0}^{Y} \hookrightarrow F_{m}^{Y}$, the space $A$ is canonically embedded in $F_{0}$ and hence in $F_{i} \supset F_{0}$ for any $i \geq 0$.

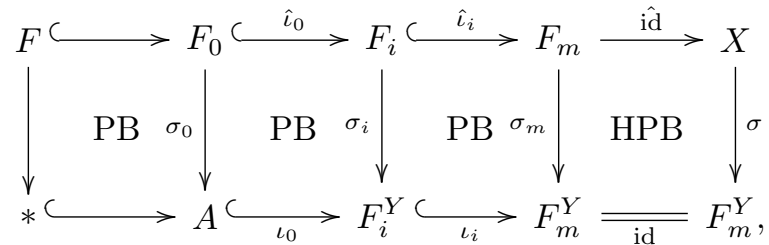

where $F$ denotes the homotopy fibre of $\sigma$ and $F_{m}$ is the homotopy pullback of $\sigma$ and the identity of $F_{m}^{Y}$. Since $\rho \circ \sigma \sim 1_{X},\left.\rho\right|_{F_{i}^{Y}}$ can be compressed into $F_{i}$ and we have the 
following commutative diagram:

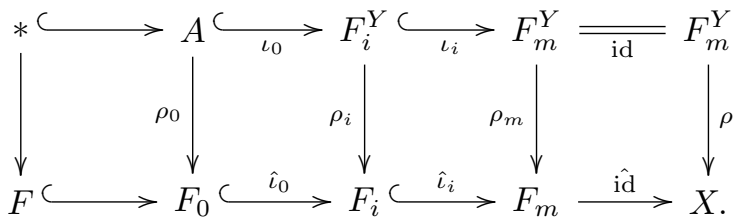

Then by the definition of categorical sequence, there is a compression $\nu_{i}^{Y}: F_{i}^{Y} \rightarrow F_{m}^{Y} \times *$ $\cup F_{i-1}^{Y} \times F_{m}^{Y}$ of the diagonal map $\Delta_{F_{i}^{Y}}: F_{i}^{Y} \rightarrow F_{i}^{Y} \times F_{i}^{Y} \subseteq F_{m}^{Y} \times F_{m}^{Y}$ relative to $F_{i-1}^{Y}$ :

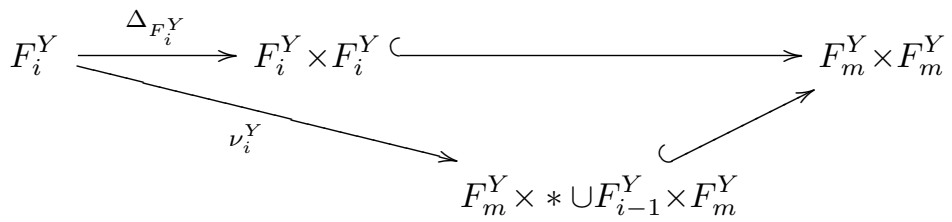

By composing $\rho_{i}$ and $\sigma_{i}$, we obtain a compression of the diagonal map $\Delta_{F_{i}}: F_{i} \rightarrow$ $F_{i} \times F_{i} \subseteq F_{m} \times F_{m}$ as follows:

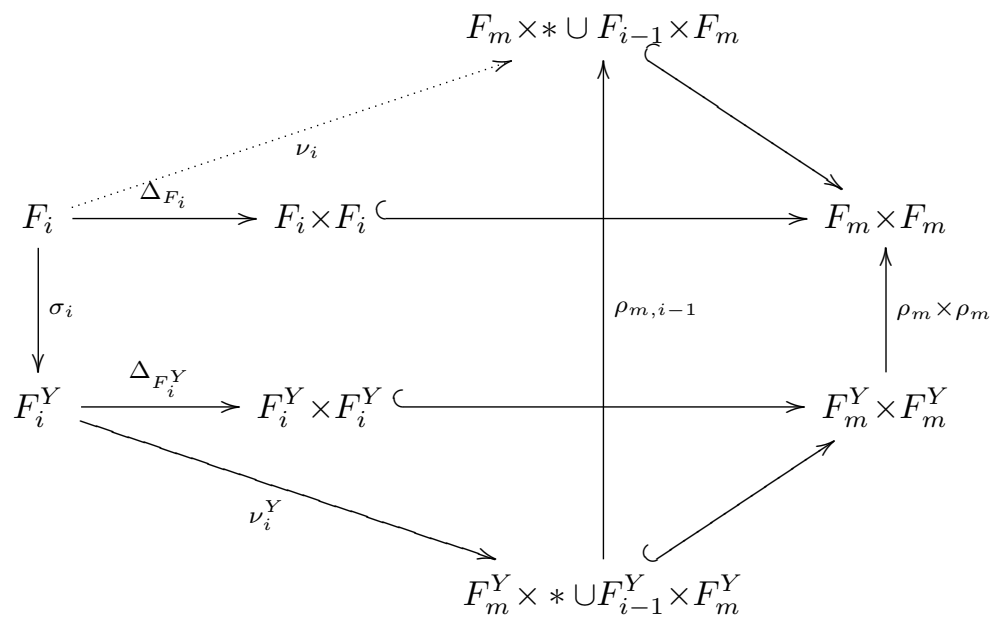

This implies $\operatorname{cat}\left(X^{\prime} ; X^{\prime}, F_{m-1}^{X}: A\right) \leq 1$, and hence $X^{\prime}=F_{m}^{X} \supset F_{m-1} \supset \cdots \supset F_{0}=A$ gives a categorical sequence for $X$.

The following lemma is our version of the result of Arkowitz and Lupton [1]:

Lemma 5.2. Let $X$ be a space in $\mathcal{T}$ with cat $(X)=m$ and $\left\{F_{i} ; 0 \leq i \leq m\right\}$ be a categorical sequence for $X$. Then there is a map $\mu: F_{i} \rightarrow F_{m} / F_{i-1} \vee F_{m}$ in $\mathcal{T}$ with axes $F_{i} \rightarrow$ $F_{m} / F_{i-1}$ and the inclusion $F_{i} \hookrightarrow F_{m}$.

Proof. By the definition of a categorical sequence, the diagonal map $\Delta: F_{i} \rightarrow F_{i} \times F_{i} \subseteq$ $F_{m} \times F_{m}$ is compressible into $F_{i-1} \times F_{m} \cup F_{m} \times *$ as $F_{i} \stackrel{\hat{\mu}}{\rightarrow} F_{i-1} \times F_{m} \cup F_{m} \times * \subseteq F_{m} \times F_{m}$. Since $F_{m} / F_{i-1} \vee F_{m}$ can be regarded as the pushout of the second projection $\operatorname{pr}_{2}$ : $F_{i-1} \times F_{m} \rightarrow F_{m}$ and the canonical inclusion $\iota: F_{i-1} \times F_{m} \hookrightarrow F_{i-1} \times F_{m} \cup F_{m} \times *$, we have 
the following diagram:

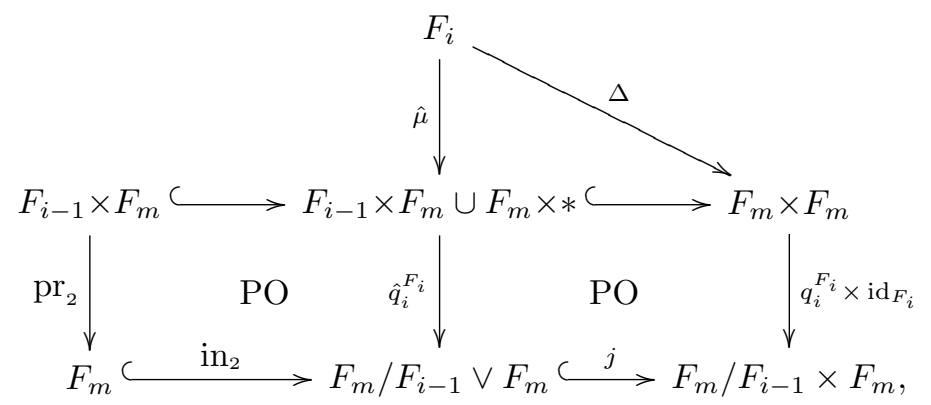

where $q_{i}^{F_{i}}: F_{i} \rightarrow F_{i} / F_{i-1} \subseteq F_{m} / F_{i-1}$ denotes the canonical collapsing map in $\mathcal{T}$. Let $\mu$ be the composition $\hat{q}_{i}^{F_{i}} \circ \hat{\mu}: F_{i} \rightarrow F_{i} / F_{i-1} \vee F_{m}$ so that $j \circ \mu$ is homotopic to $\left(q_{i}^{F_{i}} \times \operatorname{id}_{F_{i}}\right) \circ \Delta$. Thus $\mu$ has axes $q_{i}^{F_{i}}: F_{i} \rightarrow F_{i} / F_{i-1} \subseteq F_{m} / F_{i-1}$ and the inclusion $F_{i} \hookrightarrow F_{m}$.

From this one immediately deduces corollary 1.17 of the introduction.

6. Cup length and module weight for the relative L-S category. A computable lower estimate in L-S category theory is given by the classical cup-length. Here we give the definition for our new relative L-S category.

Definition 6.1. For any two maps $f:(L: A) \subset(X: A)$ and $g:(K: A) \rightarrow(X: A)$ in $\mathcal{T}^{A}$, we define cup length for $(g, f)=(X ; K, L: A)$ :

(1) Let $h$ be a multiplicative generalized cohomology theory.

$$
\operatorname{cup}(g, f ; h)=\operatorname{Min}\left\{\begin{array}{l|l}
m \geq 0 & \begin{array}{l}
\forall\left\{v_{0} \in h^{*}(X, L) ; v_{1}, \cdots, v_{m} \in h^{*}(X, A)\right\} \\
g^{*}\left(v_{0} \cdot v_{1} \cdots v_{m}\right)=0 \text { in } h^{*}(K, A)
\end{array}
\end{array}\right\} .
$$

(2) $\operatorname{cup}(g, f)=\operatorname{Max}\left\{\begin{array}{l|l}\operatorname{cup}(g, f ; h) & \begin{array}{l}h \text { is a multiplicative generalized } \\ \text { cohomology theory }\end{array}\end{array}\right\}$.

Then we have $\operatorname{cup}(g, f ; h) \leq \operatorname{cup}(g, f) \leq \operatorname{cat}(g, f)$ for any multiplicative generalized cohomology $h$. When $h$ is the ordinary cohomology with a coefficient ring $R$, we denote $\operatorname{cup}(g, f ; h)$ by $\operatorname{cup}(g, f ; R)$. This definition immediately implies the following.

REMARK 6.2. For $(g, f)=(X ; K, L: A)$, using the $\operatorname{arguments}$ in [16], we have $\operatorname{cup}(g, f)=$ $\operatorname{Min}\left\{m \geq 0 \mid \tilde{\Delta}_{K}^{m+1}: K / A \rightarrow X / L \wedge \bigwedge^{m} X / A\right.$ is stably trivial $\}$.

Let us recall that Rudyak [23] and Strom [26] introduced a homotopy theoretical version of Fadell-Husseini's category weight (see [6]). But unfortunately, we have not been able to give a version of category weight for our new relative L-S category. In this paper, we give instead a version of module weight which is a better computable lower estimate for our relative L-S category than cup length: let $f:(L: A) \subset(X: A)$ and $g:(K: A) \rightarrow(X: A)$ be maps in $\mathcal{T}^{A}$ and let $h$ be a generalized cohomology theory.

Definition 6.3 ([16]). A homomorphism $\phi: h^{*}(Y, L) \rightarrow h^{*}(K, A)$ of $h_{*}$-modules is called an (unstable) $h$-morphism if it preserves the action of any (unstable) cohomology operation on $h^{*}$. 
Definition 6.4. An (unstable) module weight $\operatorname{Mwgt}(g, f ; h)$ of $(g, f)$ with respect to $h$ is defined as follows.

$$
\operatorname{Mwgt}(g, f ; h)=\operatorname{Min}\left\{\begin{array}{l|l}
m \geq 0 & \begin{array}{l}
\text { There is a (unstable) } h \text {-morphism } \phi: \\
h^{*}\left(P^{m}(\Omega(X, L)), L\right) \rightarrow h^{*}(K, A) \text { such that } \\
\phi \circ\left(e_{m}^{X}\right)^{*}=g^{*}: h^{*}(X, L) \rightarrow h^{*}(K, A) .
\end{array}
\end{array}\right\} .
$$

When $h$ is the ordinary cohomology theory with coefficients in a ring $R$, we denote $\operatorname{Mwgt}(g, f ; h)$ by $\operatorname{Mwgt}(g, f ; R)$.

REMARK 6.5. The invariants introduced in this paper satisfy the following inequality for any generalised cohomology theory $h^{*}$ :

$$
\operatorname{cup}(g, f ; h) \leq \operatorname{Mwgt}(g, f ; h) \leq \operatorname{cat}(g, f)=\operatorname{catlen}(g, f),
$$

and hence for any ring $R$, we have

$$
\operatorname{cup}(g, f ; R) \leq \operatorname{Mwgt}(g, f ; R) \leq \operatorname{cat}(g, f)=\operatorname{catlen}(g, f) .
$$

Similar to the above definition of $\operatorname{cup}(g, f)$, we define the following invariants.

Definition 6.6. For any $(g, f)=(X ; K, L: A)$, we define

$$
\operatorname{Mwgt}(g, f)=\operatorname{Max}\left\{\begin{array}{l|l}
m \geq 0 & \begin{array}{l}
\operatorname{Mwgt}(g, f ; h)=m \text { for some generalized } \\
\text { cohomology theory } h
\end{array}
\end{array}\right\} .
$$

REMARK 6.7. $\operatorname{cup}(g, f) \leq \operatorname{Mwgt}(g, f) \leq \operatorname{cat}(g, f)=\operatorname{catlen}(g, f)$.

7. Examples of categorical sequences. In [3], Berstein and Hilton showed that the L-S category of the cell complex $Q(\alpha)=S^{r} \cup_{\alpha} e^{q+1}, \alpha \in \pi_{q}\left(S^{r}\right)$, is determined by the Hopf invariant $H_{1}(\alpha) \in \pi_{q+1}\left(S^{r} \times S^{r}, S^{r} \vee S^{r}\right)\left(\cong \pi_{q}\left(\Omega\left(S^{r}\right) * \Omega\left(S^{r}\right)\right)\right.$ by Ganea). We can easily observe that $F_{0}=*, F_{1}=S^{r}$ and $F_{2}=Q(\alpha)$ give a cone decomposition of $Q(\alpha)$ of length 2 . If $H_{1}(\alpha)=0$, then by Theorem 1.15 , we obtain that $F_{0}^{\prime}=F_{0}=*$, $F_{1}^{\prime}=F_{1} \cup_{\alpha} e^{q+1}=F_{2}=Q(\alpha)$ give a categorical sequence of length 1 .

In [15], the author showed that the L-S category of total space $E(\beta)=Q(\beta) \cup_{\psi(\beta)}$ $e^{q+r+1}, \beta \in \pi_{q}\left(S^{r}\right), \psi(\beta) \in \pi_{q+r}(Q(\beta))$ is determined by $\Sigma^{r} H_{1}(\beta) \in \pi_{q+r}\left(\Omega\left(S^{r}\right) *\right.$ $\Omega(Q(\beta)) * \Omega(Q(\beta)))$, if $H_{1}(\beta) \neq 0$. We can easily observe that $F_{0}=*, F_{1}=S^{r}, F_{2}=Q(\beta)$ and $F_{3}=E(\beta)$ give a cone decomposition of $E(\beta)$ of length 3 . If $\Sigma^{r} H_{1}(\alpha)=0$, then by Theorem 1.15, we obtain that $F_{0}^{\prime}=F_{0}=*, F_{1}^{\prime}=F_{1}=S^{r}, F_{2}^{\prime}=F_{2} \cup_{\psi(\beta)} e^{q+r+1}=F_{3}=$ $E(\beta)$ give a categorical sequence of length 2 .

Let us denote by $Z^{(k)}$ the $k$-skeleton of a CW complex $Z$. To give an upper-bound for L-S category of the total space of a fibre bundle $F \hookrightarrow E \rightarrow B$, we need a refinement of results of Varadarajan [28] and Hardie [11], and the corresponding result for strong category of Ganea [9]:

Theorem $7.1([28,11,9]) . \quad(1) \operatorname{cat}(E)+1 \leq(\operatorname{cat}(F)+1) \cdot(\operatorname{cat}(B)+1)$.

(2) $\operatorname{Cat}(E)+1 \leq(\operatorname{Cat}(F)+1) \cdot(\operatorname{Cat}(B)+1)$.

In [18], Iwase-Mimura-Nishimoto gave a refinement in the case when the base space $B$ is non-simply connected. On the other hand in the case when $B$ is simply connected, Iwase-Kono [17] gave another refinement if the higher Hopf invariant of the characteristic map is 0 . 
By assuming the fibre $F$ is of categorical length $m$, we obtain a further refinement using categorical sequence in place of cone decomposition:

Theorem 7.2. Let $B$ be a $(d-1)$-connected finite dimensional $C W$ complex $(d \geq 1)$, whose cells are concentrated in dimensions $0,1, \ldots, s \bmod d$ for some $s, 0 \leq s \leq d-1$. Let $F \hookrightarrow X \rightarrow B$ be a fibre bundle with fibre $F$ whose structure group is a compact Lie group $G$. Then we have $\operatorname{cat}(X) \leq m+\left[\frac{\operatorname{dim} B}{d}\right]$, if $F$ has a categorical sequence of length $m$ with the following compatibility assumption for some $d \geq 1$ :

(1) $\left.\psi\right|_{G^{(d \cdot(i+1)+s-1)} \times F_{j}}: G^{(d \cdot(i+1)+s-1)} \times F_{j} \rightarrow F$ is compressible into $F_{i+j}, 0 \leq i, j \leq$ $i+j \leq m$.

In [17], Kono and the author showed that there is a cone decomposition $E_{i}, 0 \leq i \leq 8$ and $E_{8}^{\prime}$ of $\operatorname{Spin}(9)$ of length 9 , while the L-S category of Spin(9) is 8 by a combination of a higher Hopf invariant and the cone decomposition: we can easily see that the construction in $\S 1$ in [17] gives the following proposition:

Proposition 7.3. Let $G \hookrightarrow E \rightarrow \Sigma^{2} V$ be a principal bundle with a characteristic map $\alpha: \Sigma V \rightarrow Q$ a subspace of $G$. Then catlen $(E) \leq m+n+1$ if $G$ has a categorical sequence $*=F_{0} \subset \cdots \subset F_{m} \simeq G$ with the following compatibility assumption for a positive integer $n$ :

(1) the restriction of the multiplication $\mu: G \times G \rightarrow G$ to the subspace $F_{j} \times Q \subseteq F_{m} \times F_{m}$ $\simeq G \times G$ is compressible into $F_{j+n} \subseteq F_{m} \simeq G, j \geq 0$ as $\mu_{j}: F_{j} \times Q \rightarrow F_{j+n}$ such that $\left.\mu_{j}\right|_{F_{j-1} \times Q}=\mu_{j-1}$.

REMARK 7.4. If we choose $n=m$, then the assumption (1) above is automatically satisfied and we always have $\operatorname{cat}(E) \leq 2 \operatorname{cat}(G)+1$ which is a special case of a theorem of Hardie and Varadarajan [11, 28] (see Theorem 7.1 (1)).

Moreover, Lemma 1.1 in [17] implies that the higher Hopf invariant of the attaching map of the top cell of $\operatorname{Spin}(9)$ must vanish, since the structure map of cat $\left(E_{8}^{\prime}\right)=8$ can be chosen to be compatible to the structure map of $\operatorname{cat}\left(E_{8}\right)=8$ by the argument given in the proof of Lemma 1.1 in [17]. Hence by Theorem 1.15 , we obtain that $E_{i}, 0 \leq i \leq 7$ and $E_{8}^{\prime}$ give the categorical sequence of length 8: we can easily see that the proof of Lemma 1.1 in [17] gives the following theorem:

Proposition 7.5. Let $G \hookrightarrow E \rightarrow \Sigma^{2} V$ be a principal bundle with a characteristic map $\alpha: \Sigma V \rightarrow Q$ a subspace of $G$. Then $\operatorname{cat}(E) \leq \operatorname{Max}\{m+n, m+2\}$ if $G$ has a categorical sequence $*=F_{0} \subset \cdots \subset F_{m} \simeq G$ with the following compatibility assumptions for a positive integer $n$ :

(1) the restriction of the multiplication $\mu: G \times G \rightarrow G$ to the subspace $F_{j} \times Q \subseteq F_{m} \times F_{m}$ $\simeq G \times G$ is compressible into $F_{j+n} \subseteq F_{m} \simeq G, j \geq 0$ as $\mu_{j}: F_{j} \times Q \rightarrow F_{j+n}$ such that $\left.\mu_{j}\right|_{F_{j-1} \times Q}=\mu_{j-1}$ and

(2) $H_{n}^{\left(E ; Q \cup_{\alpha} C \Sigma V, Q ; *\right)}(\alpha)=0$.

These propositions imply the following result.

ThEOREM 7.6. Let $G \hookrightarrow E \rightarrow \Sigma^{2} V$ be a principal bundle with a characteristic map $\alpha: \Sigma V \rightarrow Q$, a subspace of $G$. Then $\operatorname{cat}(E) \leq \operatorname{Max}\left\{m+n+\operatorname{cat}\left(E ; Q \cup_{\alpha} C \Sigma V, Q ; *\right), m+2\right\}$ 
if $G$ has a categorical sequence $*=F_{0} \subset \cdots \subset F_{m} \simeq G$ with the following compatibility assumption for a positive integer $n \geq 1$ :

(1) the restriction of the multiplication $\mu: G \times G \rightarrow G$ to the subspace $F_{j} \times Q \subseteq F_{m} \times F_{m}$ $\simeq G \times G$ is compressible into $F_{j+n} \subseteq F_{m} \simeq G, j \geq 0$ as $\mu_{j}: F_{j} \times Q \rightarrow F_{j+n}$ such that $\left.\mu_{j}\right|_{F_{j-1} \times Q}=\mu_{j-1}$.

Acknowledgments. Research of the author is supported by the Grant-in-Aid for Scientific Research \#15340025 from Japan Society for the Promotion of Science.

\section{References}

[1] M. Arkowitz and G. Lupton, Homotopy actions, cyclic and their duals, Homology, Homotopy and Applications 7 (2005), 169-184.

[2] I. Berstein and T. Ganea, The category of a map and of a cohomology class, Fund. Math. 50 (1961/62), 265-279.

[3] I. Berstein and P. J. Hilton, Category and generalised Hopf invariants, Illinois J. Math. 12 (1968), 421-432.

[4] O. Cornea, Some properties of the relative Lusternik-Schnirelmann category, in: Stable and Unstable Homotopy (Toronto, 1996), Fields Inst. Commun. 19, 1998, 67-72.

[5] L. Fernández-Suárez, A. Gómez-Tato, J. Strom and D. Tanré, The Lusternik-Schnirelmann category of Sp(3), Proc. Amer. Math. Soc. 132 (2004), 587-595.

[6] E. Fadell and S. Husseini, Category weight and Steenrod operations, Papers in honor of José Adem, Bol. Soc. Mat. Mexicana 37 (1992), 151-161.

[7] E. Fadell and S. Husseini, Relative category, products and coproducts, Rend. Sem. Mat. Fis. Milano 64 (1994), 99-115 (1996).

[8] R. H. Fox, On the Lusternik-Schnirelmann category, Ann. of Math. (2) 42 (1941), 333-370.

[9] T. Ganea, Lusternik-Schnirelmann category and strong category, Illinois J. Math. 11 (1967), 417-427.

[10] T. Ganea, Cogroups and suspensions, Invent. Math. 9 (1970), 185-197.

[11] K. A. Hardie, A note on fibrations and category, Michigan Math. J. 17 (1970), 351-352.

[12] J. Harper, A proof of Gray's conjecture, in: Algebraic Topology (Evanston, 1988), Contemp. Math, 96, Amer. Math. Soc., Providence, 1989, 189-195.

[13] N. Iwase, Ganea's conjecture on Lusternik-Schnirelmann category, Bull. London Math. Soc. 30 (1998), 623-634.

[14] N. Iwase, $A_{\infty}$-method in Lusternik-Schnirelmann category, Topology 41 (2002), 695-723.

[15] N. Iwase, Lusternik-Schnirelmann category of a sphere-bundle over a sphere, Topology 42 (2003), 701-713.

[16] N. Iwase, The Ganea conjecture and recent developments on the Lusternik-Schnirelmann category, Sūgaku 56 (2004), 281-296 (in Japanese).

[17] N. Iwase and A. Kono, Lusternik-Schnirelmann category of Spin(9), Trans. Amer. Math. Soc. 359 (2007), 1517-1526.

[18] N. Iwase, M. Mimura, T. Nishimoto, L-S categories of non-simply-connected compact simple Lie groups, Topology Appl. 150 (2005), 111-123.

[19] I. M. James, The Topology of Stiefel Manifolds, London Math. Soc. Lec. Notes 24, Cambridge University Press, Cambridge, 1976. 
[20] I. M. James, On category, in the sense of Lusternik-Schnirelmann, Topology 17 (1978), 331-348.

[21] I. M. James, Lusternik-Schnirelmann category, in: Handbook of Algebraic Topology, North Holland, Amsterdam, 1995, 1293-1310.

[22] L. Lusternik and L. Schnirelmann, Méthodes Topologiques dans les Problèmes Variationnels, Hermann, Paris, 1934.

[23] Y. B. Rudyak, On category weight and its applications, Topology 38 (1999), 37-55.

[24] M. Sakai, A proof of the homotopy push-out and pull-back lemma, Proc. Amer. Math. Soc. 129 (2001), 2461-2466.

[25] J. D. Stasheff, Homotopy associativity of H-spaces, I, II, Trans. Amer. Math. Soc. 108 (1963), 275-292, 293-312.

[26] J. Strom, Essential category weight and phantom maps, in: Cohomological Methods in Homotopy Theory (Bellaterra, 1998), Progr. Math. 196, Birkhäuser, Basel, 2001, 409-415.

[27] J. Strom, Decomposition of the diagonal map, Topology 42 (2003), 349-364.

[28] K. Varadarajan, On fibrations and category, Math. Z. 88 (1965), 267-273.

[29] G. W. Whitehead, Elements of Homotopy Theory, Graduate Texts in Mathematics 61, Springer, Berlin, 1978. 